\title{
A nonlinear oblique derivative boundary value problem for the heat equation Part 1: Basic results
}

by

\author{
Florian MEHATS \\ Centre de Mathématiques Appliquées URA CNRS 756 \\ École Polytechnique, 91128 Palaiseau Cedex, France \\ and \\ Jean-Michel ROQUEJOFFRE \\ UFR-MIG, Université de Toulouse III UMR CNRS 5640 \\ 118, route de Narbonne, 31062 Toulouse Cedex, France
}

ABSTRACT. - We study the heat equation $B_{t}-\Delta B=0$ in the halfplane with the nonlinear oblique derivative condition $B_{X}=K B B_{Z}$ on the boundary, where $\left(B_{X}, B_{Z}\right)$ are respectively the normal and the tangential derivatives of $B$. The ultimate goal is to let $K \rightarrow+\infty$ in the equations.

In this first part, we introduce self-similar solutions which verify an elliptic equation with the same nonlinear boundary condition. The main part of this first paper concerns this self-similar problem. It is well-posed and its solution is shown to be smooth, by means of boundary integral estimates. The originality of the approach is the robustness of the estimates with respect to $K$. The evolution problem itself admits global classical solutions which converge, as times tends to $+\infty$, to the self-similar solution.

(C) 1999 L'Association Publications de l'Institut Henri Poincaré. Published by Elsevier B.V. All rights reserved

Key words: Nonlinear oblique derivative condition, heat equation, self-similar solution.

RÉsumé. - Nous étudions l'équation de la chaleur $B_{t}-\Delta B=0$ dans le demi-plan, avec la condition aux limites $B_{X}=K B B_{Z}$ sur la frontière. Il s'agit une condition oblique non linéaire, $B_{X}$ et $B_{Z}$ étant respectivement

Annales de l'Institut Henri Poincaré - Analyse non linéaire - 0294-1449

Vol. 16/99/02/

(C) 1999 L'Association Publications de l'Institut Henri Poincaré. Published by Elsevier B.V. All rights reserved 
les dérivées normale et tangentielle de $B$ sur cet axe. Notre but final est de faire tendre $K$ vers $+\infty$ dans les équations.

Ce travail est divisé en deux parties. Dans cette première partie, nous introduisons des solutions autosemblables associées à ce problème.

Ces solutions autosemblables vérifient un système elliptique, avec la même condition aux limites non linéaire sur l'axe, et l'étude de ce problème constitue la majeure partie de ce premier article. Nous prouvons, au moyen d'estimations intégrales à la frontière, qu'il est bien posé et que sa solution est régulière. L'apport de cette approche est la robustesse des estimations vis-à-vis de $K$. Le problème d'évolution proprement dit admet des solution classiques, qui convergent en temps vers la solution autosemblable.

(C) 1999 L'Association Publications de l'Institut Henri Poincaré. Published by Elsevier B.V. All rights reserved

\section{INTRODUCTION AND MAIN RESULTS}

This paper is the first of a series aimed at studying the long-time behaviour of a simple nonlinear oblique boundary value problem, written as follows.

$$
\begin{cases}B_{t}-\Delta B=0 & \left(\mathbb{R}_{+}^{2}\right) \\ B_{X}-K B B_{Z}=0 & (X=0) \\ B(t,-\infty, X)=1, \quad B(t,+\infty, X)=0 & \end{cases}
$$

where $B(t, Z, X) \in \mathbb{R}$,

$\mathbb{R}_{+}^{2}=\left\{(Z, X) \in \mathbb{R} \times \mathbb{R}_{+}\right\}$,

$K>0$.

Our eventual goal is to let $K \rightarrow+\infty$ in these equations.

This system occurs in plasma physics and describes the penetration of a magnetic field in a plasma along a perfectly conducting electrode.

Let a plasma be injected in $\mathbb{R}_{+}^{2}$ through an anode located on the axis $\{X=0\}$. The cathode will be ignored in this paper; indeed, we assume that if it is far enough it does not perturb the phenomenon we are interested in here, which occurs near the anode. This plasma is a fluid composed of electrons and ions. In this problem, we consider the magnetic field dynamics during the propagation of an electromagnetic wave in this medium. This propagation is modified by the presence of the light electron component in the medium. This phenomenon occurs in devices such as plasma switches ([6], [9]). 
The domain is supposed here to be infinite $\left(R_{+}^{2}\right)$ since our aim is to study self-similar solutions. After the instant $t=0$ we apply a constant magnetic field at $z=-\infty$ (the entry of the subset).

The derivation of the model is made in the frame of the Electron Magnetohydrodynamics ([9], [10]): this is a fluid model derived from Magnetohydrodynamics where we neglect the motion of ions and the inertia of electrons. The characteristics of the plasma are constant during the time and, for the sake of simplicity ([8]), we assume that its density $n$ is uniform. Therefore, the propagation of the field will only be diffusive. Moreover, we assume that the magnetic field has only a component orthogonal to $\mathbb{R}_{+}^{2}$, denoted $\mathcal{B}$.

The electric field $\mathcal{E}$ can be expressed in function of $\mathcal{B}$ and, finally, we can reduce all the equations of our model to the following one, governing the unknown $\mathcal{B}$.

The conservation of momentum for the electrons coupled with Maxwell equations gives a scalar equation on $\mathcal{B}(t, Z, X)$ :

$$
\mathcal{B}_{t}-\frac{1}{\mu_{0} \sigma} \Delta \mathcal{B}=0, \quad \forall(t, Z, X) \in \mathbb{R}_{+}^{*} \times \mathbb{R}_{+}^{2},
$$

where $\mu_{0}$ is the permeability of free space

$\sigma$ is the conductivity of the plasma (isotropic and uniform here).

At $Z \rightarrow-\infty$ we have the source of magnetic field $\mathcal{B}=\mathcal{B}_{0}(\forall t \geq 0)$. The equation (1.2) describes the diffusive penetration of $\mathcal{B}$ in the plasma if we let the dimensions tend to the infinity. The key point here is the influence of the electrode, through which the plasma is injected. Since it is a perfect conductor, on $X=0$ the electric field $\mathcal{E}$ is orthogonal to the surface so it follows from the relation between $\mathcal{E}$ and $\mathcal{B}$

$$
\mathcal{B}_{X}-\frac{\sigma}{e n} \mathcal{B B}_{Z}=0, \quad \text { for } X=0 \text { and } \forall(t, Z) \in \mathbb{R}_{+}^{*} \times \mathbb{R} .
$$

Next we rescale the equations (1.2)-(1.3) to obtain the parabolic system $(1.1 \mathrm{NH})$ of $B=\mathcal{B} / \mathcal{B}_{0}$, with the non dimensional constant $K=\frac{\sigma \mathcal{B}_{0}}{\text { en }}$. Formal studies of this system were made by the authors of [8] in the case where the diffusion along $z$ is suppressed.

In the general study of quasilinear parabolic systems, much is known. In [2] and [3], a general abstract framework is established, and yields local existence theorems and continuation criteria. In [13], very general smoothness results for nonlinear elliptic oblique boundary value problems in Vol. 16, $\mathrm{n}^{\circ} 2-1999$. 
bounded domains are given; they include in particular the $B_{X}-K B B_{Z}=0$ boundary condition. In [7], [15], global existence theorems are proved for parabolic equations with fully nonlinear oblique conditions are also proved, in bounded domains.

The smoothness arguments of [7], [15] being local, we could rely on them for smoothness results in our context, and this is what we are going to do for the Cauchy Problem. As for the self-similar solutions, the coefficients of the equation are unbounded; we could still rely on [13], at the expense of keeping track of the coefficients in the estimates. We shall instead present an alternative approach, based on the conservative form of the boundary condition; this will yield immediate $H^{3 / 2}$ estimates. Thus there is enough regularity to allow, via a simple sub- and super-solution method, the construction ex nihilo of the self-similar solutions. It is at this stage quite tempting to find out whether this idea is sufficient to prove further smoothness, with the sole help of the classical Agmon-Douglis-Nirenberg estimates [1]. We therefore explore it further, and the scheme turns out to be as follows: $H^{3 / 2}$ will imply $W^{2 / 3.3}$, which will in turn imply $H^{2}$. A blowup argument will lead us to Lipschitz, which will in turn imply $C^{1, \alpha}$. At this stage, the classical boundary Hölder estimates [1], [12] are applicable.

To sum up, our contribution in this first part are the following:

- treatment of a nonlinear oblique derivative problem with unbounded coefficients in an infinite domain,

- a natural smoothness proof, which is quite simple - at least in its early stages,

- robust estimates with respect to $K$. Indeed, a remarkable fact of this approach is that it lays the basis for much more degenerate equations; in particular the boundary integral estimates may be rendered independent of $K$ by rescaling. This will be exploited in the second part [14].

The self-similar problem linked to $(1.1 \mathrm{NH})$ reads, in the reduced variables $z=\frac{Z}{\sqrt{t+1}}$ and $x=\frac{X}{\sqrt{t+1}}$,

$(1.4 \mathrm{NH})$

$$
\begin{cases}-U_{x x}-\frac{1}{2}\left(z U_{z}+x U_{x}\right)=0 & \left(\mathbb{R}_{+}^{2}\right) \\ U_{x}-K U U_{z}-0 & (x=0) \\ U(-\infty, x)=1, U(+\infty, x)=0 . & \end{cases}
$$

We shall denote by $\psi(z)$ the solution of $(1.4 \mathrm{H})$ corresponding to $K=0$.

The main results of this first part are contained in the following two theorems.

THEOREM 1.1. - There exists a unique solution $U \in C^{\infty}\left(\mathbb{R}_{+}^{2}\right)$ of $(1.4 \mathrm{NH})$. Moreover we have the following properties: 
- $\exists C>0$ such that $0 \leq U(z, x)-\psi(z) \leq C \exp \left(-\frac{z^{2}+x^{2}}{8}\right)$

- $U$ is decreasing with respect to $z$ and $x$

THEOREM 1.2. - Let $B_{0} \in C\left(\mathbb{R}_{+}^{2}\right)$ be such that $B_{0}-U$ tends to 0 (fast enough) as $|(x, z)| \rightarrow+\infty$.

The Cauchy Problem for (1.1NH) has a unique classical solution $B(t, X, Z)$. There holds

$$
\lim _{t \rightarrow+\infty} \sup _{X^{-}, Z}\left|B(t, X, Z)-U\left(\frac{X}{\sqrt{t+1}}, \frac{Z}{\sqrt{t+1}}\right)\right|=0 .
$$

Moreover, if $\frac{B_{0}-U}{|\nabla U|}$ tends to 0 as $|(x, z)| \rightarrow+\infty$, there holds, for all $\alpha>0$,

$$
\sup _{X, Z}\left|B(t, X, Z)-U\left(\frac{X}{\sqrt{t+1}}, \frac{Z}{\sqrt{t+1}}\right)\right|=O\left(t^{-1 / 2+\alpha}\right) .
$$

In the following two sections, we study the solution of $(1.4 \mathrm{H})$. Its existence in $H^{3 / 2}\left(\mathbb{R}_{+}^{2}\right)$ is proved in Section 2, and we explain in Section 3 how we may work our way through to $C^{\infty}$ regularity. Theorem 1.2 is proved in Section 4.

\section{CONSTRUCTION OF THE SELF-SIMILAR SOLUTION IN $H^{3 / 2}$ AND UNIQUENESS}

To avoid inhomogeneous boundary condition at $Z \rightarrow+\infty$, we introduce the function $\beta(t, Z)$ solution of the one-dimensional heat equation

$$
\left\{\begin{array}{l}
\frac{\partial \beta}{\partial t}-\frac{\partial^{2} \beta}{\partial Z^{2}}=0 \\
\beta(t,-\infty)=1, \quad \beta(t,+\infty)=0 \\
\beta(t=0)=\frac{1}{2 \sqrt{\pi}} \int_{Z}^{+\infty} e^{-\frac{\sigma^{2}}{4}} d \sigma
\end{array}\right.
$$

This choice of the initial condition for $\beta$ is not very important for the study of this evolution problem but is made here to be compatible further with the self-similar problem and with the function $\psi$. There holds

$$
\beta(t, Z)=\frac{1}{2 \sqrt{\pi}} \int_{Z / \sqrt{t+1}}^{+\infty} e^{-\frac{\sigma^{2}}{4}} d \sigma .
$$

Vol. 16, $\mathrm{n}^{\circ}$ 2-1999. 
We will say that $B$ is the solution of $(1.1 \mathrm{NH})$ if $b=B-\beta$ is homogeneous at the infinity and is solution of

$$
\begin{cases}b_{t}-\Delta b=0 & \left(\mathbb{R}_{+}^{2}\right) \\ b_{X}-K(b+\beta)(b+\beta)_{Z}=0 & (X=0) \\ b(t, Z, X) \rightarrow 0 \text { if }|(Z, X)| \rightarrow+\infty & \end{cases}
$$

We have already introduced the function $\psi(z)$ from the real variable, solution of

$$
\left\{\begin{aligned}
-\psi_{z z} & -\frac{1}{2} z \psi_{z}=0 \\
\psi(-\infty) & =1 ; \psi(+\infty)=0
\end{aligned}\right.
$$

We have the analytical expression $\psi(z)=\frac{1}{2 \sqrt{\pi}} \int_{z}^{+\infty} e^{-\frac{\sigma^{2}}{4}} d \sigma$ and $\psi(z)=\beta(t, Z)$. Moreover $u=U-\psi$ verifies

$$
\begin{cases}-\Delta u-\frac{1}{2}\left(z u_{z}+x u_{x}\right)=0 & \left(\mathbb{R}_{+}^{2}\right) \\ u_{x}-K(u+\psi)(u+\psi)_{z}=0 & (x=0) \\ u \rightarrow 0 \text { if }|(z, x)| \rightarrow+\infty & \end{cases}
$$

The plan of this section is the following: we first regularize the system $(1.4 \mathrm{H})$. We prove that this new problem has a solution, then with a priori estimates we can pass to the limit. Finally we prove the uniqueness.

\subsection{Regularized problem}

If $v \in H^{1}\left(\mathbb{R}_{+}^{2}\right)$, we denote by $\gamma v$ its trace on the axis $x=0$ (sometimes $v$ when there is no ambiguity). Then we introduce a regularizing operator $T^{\varepsilon}$ from $H^{1 / 2}(\mathbb{R})$ to $C^{1, \alpha}(\mathbb{R})$, where $0<\alpha<1$ :

Let $v \in H^{1 / 2}(\mathbb{R})$ and $V(t, z)$ bc the solution of

$$
\left\{\begin{array}{l}
V(0, z)=v(z) \\
V_{t}-V_{z z}-\frac{1}{2} z V_{z}=0
\end{array}\right.
$$

then $T^{\varepsilon} v:=V(\varepsilon, z)$. Thus $T^{\varepsilon}(\gamma u)$, also denoted $T^{\varepsilon} u$, is defined as soon as $u \in H^{1}\left(\mathbb{R}_{+}^{2}\right)$ and we have the estimate:

$\left\|T^{\varepsilon}(\gamma u)\right\|_{C^{1, \alpha}(\mathbf{R})} \leq C_{\varepsilon}$, where $C_{\varepsilon}$ depends only on $\varepsilon$ and $\|u\|_{H^{1}\left(\mathbf{R}_{+}^{2}\right)}$.

Remark that, of course, $T^{\varepsilon}(v)$ is much more regular; remark also that $T^{\varepsilon}$ is linear and that $T^{\varepsilon} \psi=\psi$. This allows us to write $T^{\varepsilon}(u+\psi)=$ 
$T^{\varepsilon} u+\psi$; it is therefore equivalent to regularize the homogeneous solution (system $(1.4 \mathrm{H})$ ) or the non-homogeneous one (system $(1.4 \mathrm{H})$ ).

We define now a regularized problem associated to $(1.4 \mathrm{H})$ :

$$
\begin{cases}-\Delta u^{\varepsilon}-\frac{1}{2}\left(z u_{z}^{\varepsilon}+x u_{x}^{\varepsilon}\right)=0 & \left(\mathbb{R}_{1}^{2}\right) \\ u_{x}^{\varepsilon}-K\left(T^{\varepsilon} u^{\varepsilon}+\psi\right)\left(u^{\varepsilon}+\psi\right)_{z}=0 & (x=0) \\ u^{\varepsilon} \rightarrow 0 \text { at the infinity. } & \end{cases}
$$

We will prove the existence of a smooth $\left(C^{2, \alpha}\right)$ solution of this problem by the super-/sub-solution method. For a given positive $\varepsilon$, we have a uniform $C^{1, \alpha}$ estimate of the oblique vector $\left(-K\left(T^{\varepsilon} u^{\varepsilon}+\psi\right), 1\right)$ in the boundary condition; this will give us a solution of $(2.1 \mathrm{H})$.

Recall that $\bar{u}$ (resp. $\underline{u}$ ) is a super- (resp.sub-) solution of $(1.4 \mathrm{H})$ if and only if there holds, in the $H^{1}$ sense,

$$
\left\{\begin{array}{llc}
-\Delta \bar{u}-\frac{1}{2}\left(z \bar{u}_{z}+x \bar{u}_{x}\right) \geq 0 & (\text { resp. } \leq 0) & \left(\mathbb{R}_{+}^{2}\right) \\
\bar{u}_{x}-K(\bar{u}+\psi)(\bar{u}+\psi)_{z} \leq 0 & (\text { resp. } \geq 0) & (x=0)
\end{array}\right.
$$

Remark that 0 is an obvious sub-solution ( $\psi$ is decreasing).

LEMMA 2.1. - There exists a super-solution $\Lambda(z, x)$ of $(1.4 \mathrm{H})$ such that

$$
\forall \sigma>1 \exists C_{\sigma}>0: \quad 0 \leq \Lambda(z, x) \leq \min \left(1-\psi, C_{\sigma} \exp \left(-\frac{z^{2}+x^{2}}{4 \sigma}\right)\right)
$$

Proof. - We seek a function $\Lambda$ under the form $\Lambda(z, x)=2 \psi(x) h(z)$ with

$$
h(z)= \begin{cases}1-\psi(z) & \text { for } z \leq 0 \\ 1-\psi(z)-a z & \text { for } 0 \leq z \leq z_{1} \\ \psi\left(z-z_{0}\right) & \text { for } z_{1} \leq z\end{cases}
$$

where $z_{1}, z_{0}$ and $a$ are given. These parameters $z_{1}, z_{0}$ and $a$ will be chosen so that $\Lambda$ is continuous:

$$
1-\psi\left(z_{1}\right)-a z_{1}=\psi\left(z_{1}-z_{0}\right)
$$

A straightforward calculation shows that the first inequality of (2.2) is verified as soon as the constants $z_{1}, z_{0}$ and $a$ are positive and as

$$
h^{\prime}\left(z_{1}^{+}\right)-h^{\prime}\left(z_{1}^{-}\right) \leq 0 \text {. }
$$

This last condition comes from the $-\Lambda^{\prime \prime}$ term and from the fact that $\Lambda$ is only piecewise $C^{1}$. 
The second inequality of (2.2) (the condition on the boundary $\{x=0\}$ ) writes differently depending on the position of $z$, and we can find sufficient conditions (see the proof of Proposition 4.1 for details about the computations):

- $z \leq 0$ : this condition is automatically checked since $-\frac{1}{\sqrt{\pi}}(1-\psi) \leq 0$.

- $0 \leq z \leq z_{1}: \quad a \leq \frac{1}{2 K \sqrt{\pi}}$

(with $[C 1]$ and the convexity of $\psi$ on $\mathbb{R}_{+}$)

- $z_{1} \leq z: \begin{cases}z_{1} \geq z_{0}+2 \sqrt{\log (2 K)} & \text { if } K \geq 1 / 2 \\ z_{1}=z_{0}=0 & \text { otherwịse. }\end{cases}$

For $K \geq 1 / 2$ we can see that the following parameters are convenient to ensure $[C 1],[C 2],[C 3],[C 4]$ :

$$
\left\{\begin{array}{l}
z_{1}=4 K \sqrt{\pi} \\
z_{0}=4 K \sqrt{\pi}-2 \sqrt{\log (2 K)} \\
a \text { adjusted to ensure the continuity of } h \text { in } z_{1}, \text { i.e. [C1]. }
\end{array}\right.
$$

For $K \leq 1 / 2, h(z)$ is simpler:

$$
h(z)= \begin{cases}1-\psi(z) & \text { for } z \leq 0 \\ \psi(z) & \text { for } z \geq 0\end{cases}
$$

The estimate of $\Lambda$ stated in the lemma follows directly from the properties of $\psi(z)=\frac{1}{2 \sqrt{\pi}} \int_{z}^{+\infty} e^{-\frac{\tau^{2}}{4}} d \tau$

Write now a more general oblique derivative boundary problem, with a $C^{1, \alpha}(\mathbb{R})$ positive function $\eta(z)$ :

$$
\begin{cases}-\Delta u-\frac{1}{2}\left(z u_{z}+x u_{x}\right)=0 & \left(\mathbb{R}_{+}^{2}\right) \\ u_{x}-K \eta(u+\psi)_{z}=0 & (x=0) \\ u \rightarrow 0 \text { at the infinity. } & \end{cases}
$$

This standard linear problem has a unique $C^{2, \alpha}$ solution $u$ and we can estimate $\|u\|_{C^{2, \alpha}}$ with $\|\eta\|_{C^{1, \alpha}}$.

Let $\rho(z, x)=\exp \left(\frac{z^{2}+x^{2}}{8}\right)$. We define the usual weighted spaces

$$
\begin{gathered}
L_{\rho}^{2}\left(\mathbb{R}_{+}^{2}\right)-\left\{v \in L^{2}\left(\mathbb{R}_{+}^{2}\right) / \rho v \in L^{2}\left(\mathbb{R}_{+}^{2}\right)\right\}, \\
H_{\rho}^{1}\left(\mathbb{R}_{+}^{2}\right)=\left\{v \in L_{\rho}^{2}\left(\mathbb{R}_{+}^{2}\right) / \nabla v \in\left(L_{\rho}^{2}\left(\mathbb{R}_{+}^{2}\right)\right)^{2}\right\}
\end{gathered}
$$


and

$$
\begin{gathered}
\|v\|_{L_{\rho}^{2}\left(\mathbf{R}_{+}^{2}\right)}=\|\rho v\|_{L^{2}\left(\mathbf{R}_{+}^{2}\right)}, \\
\|v\|_{H_{\rho}^{1}\left(\mathbf{R}_{+}^{2}\right)}^{2}=\|v\|_{L_{\rho}^{2}\left(\mathbf{R}_{+}^{2}\right)}^{2}+\|\nabla v\|_{\left(L_{\rho}^{2}\left(\mathbf{R}_{+}^{2}\right)\right)^{2}}^{2} .
\end{gathered}
$$

LEMMA 2.2. - Let $u \in C^{2, \alpha}\left(\overline{\mathbb{R}_{+}^{2}}\right)$ be solution of $(2.3 \mathrm{H})$. Suppose that $\eta \leq \Lambda+\psi$. Then $u$ verifies the following properties:

(i) $0 \leq u \leq \Lambda$;

(ii) if $\eta$ is decreasing then there exists a constant $C_{0}$, independent of $\eta$ such that $\|u\|_{H_{p}^{1}\left(\mathbb{R}_{+}^{2}\right)} \leq C_{0}$;

(iii) if $\eta$ is decreasing then $u+\psi$ is decreasing with respect to $z$.

Proof. - (i). Set $w=\Lambda-u$. Since $\Lambda$ is a super-solution of $(1.4 \mathrm{H})$, we have $\Lambda_{x} \leq K(\Lambda+\psi)(\Lambda+\psi)_{z}$, for $x=0$. Moreover $(\Lambda+\psi)_{z} \leq 0$. Thus, with the assumption on $\eta$, it yields $\Lambda_{x} \leq K \eta(\Lambda+\psi)_{z}$.

Therefore $w$ verifies the inequalities

$$
\begin{cases}-\Delta w-\frac{1}{2}\left(z w_{z}+x w_{x}\right) \geq 0 & \left(\mathbb{R}_{+}^{2}\right) \\ w_{x}-K \eta(w+\psi)_{z} \leq 0 & (x=0) .\end{cases}
$$

The maximum principle coupled with Hopf's lemma implies that $w \geq 0$, since $w=0$ at the infinity. Thus $u \leq \Lambda$. The other inequality of (i) is also a consequence of the maximum principle, directly applied on $(2.3 \mathrm{H})$.

(ii). Set $\tilde{u}=\rho u$. Apply (i) and the estimate of $\Lambda$ obtained in Lemma supersolution with $\sigma=3 / 2$. There exists a constant $C>0$ such that

$$
0 \leq(1+|z|) \tilde{u} \leq C \exp \left(-\frac{z^{2}+x^{2}}{8}\right) .
$$

To estimate $u$ in the $H_{\rho}^{1}$ norm, it suffices to estimate $\tilde{u}$ in the $H^{1}$ norm. $\Lambda$ direct calculation shows that $\tilde{u}$ verifies

$$
\begin{cases}-\Delta \tilde{u}+\frac{1}{2}\left(1+\frac{1}{8}\left(z^{2}+x^{2}\right)\right) \tilde{u}=0 & \left(\mathbb{R}_{+}^{2}\right) \\ \tilde{u}_{x}-K \eta \tilde{u}_{z}=-\frac{K}{4} z \eta \tilde{u}-\frac{K}{2 \sqrt{\pi}} \eta \rho^{-1} & (x=0) .\end{cases}
$$

Multiply by $\tilde{u}$, integrate and apply Green's formula. It comes

$$
\begin{aligned}
\int_{\mathbb{R}_{+}^{2}}|\nabla \tilde{u}|^{2} & +\frac{1}{2} \int_{\mathbf{R}_{+}^{2}}\left(1+\frac{1}{8}\left(z^{2}+x^{2}\right)\right) \tilde{u}^{2} \\
& =-\int_{\mathbf{R}} \tilde{u}_{x} \tilde{u} .
\end{aligned}
$$

Vol, $16, \mathrm{n}^{\circ} 2-1999$. 
In this paper, $\int_{\mathbb{R}}$ are always integrals calculated on the axis $(z, x) \in$ $\mathbb{R} \times\{0\}$. To estimate the right-hand side of the above equality, use (2.4), (2.5) and the fact that $\eta$ is decreasing and bounded by $\Lambda+\psi \leq 1$. It follows

$$
\begin{aligned}
-\int_{\mathbb{H}} \tilde{u}_{x} \tilde{u} & =-K \int_{\mathbb{K}}\left(\eta \tilde{u}_{z} \tilde{u}-\frac{1}{4} z \eta \tilde{u}^{2}-\frac{1}{2 \sqrt{\pi}} \eta \rho^{-1} \tilde{u}\right) \\
& =K \int_{\mathbb{R}} \eta_{z}\left(\frac{\tilde{u}}{2}\right)^{2}+K \int_{\mathbb{R}} \eta\left(\frac{z}{4} \tilde{u}+\frac{1}{2 \sqrt{\pi}} \rho^{-1}\right) \tilde{u} \\
& \leq C_{1} \int_{\mathbb{R}} \tilde{u} \\
& \leq C_{2}
\end{aligned}
$$

Hence $\|\tilde{u}\|_{H^{1}\left(\boldsymbol{R}_{+}^{2}\right)} \leq C_{0}$, then $\|u\|_{H_{\rho}^{1}\left(\mathbb{R}_{+}^{2}\right)} \leq C_{0}$. This constant $C_{0}$ is independent of $\|\eta\|_{C^{1, \alpha}}$ thus of $\varepsilon$.

(iii). The solution $u$ of $(2.3 \mathrm{H})$ is in $H^{2}\left(\mathbb{R}_{+}^{2}\right)$; we are indeed in the case of a regular oblique derivative boundary problem ( $\eta$ is smooth) and the unbounded coefficients of the elliptic equation are taken in account easily after integrations by parts as in:

$$
-\int_{\mathbb{R}_{+}^{2}}\left(z u_{z}+x u_{x}\right) u-\int_{\mathbf{R}_{+}^{2}} u^{2} .
$$

Let $v=(u+\psi)_{z}$. With (ii), this function is in $C^{1, \alpha}\left(\overline{\mathbb{R}_{+}^{2}}\right) \cap H^{1}\left(\mathbb{R}_{+}^{2}\right) \cap$ $L_{\rho}^{2}\left(\mathbb{R}_{+}^{2}\right)$ and verifies

$$
\begin{cases}-\Delta v-\frac{1}{2}\left(z v_{z}+x v_{x}\right)-\frac{1}{2} v=0 & \left(\mathbb{R}_{+}^{2}\right) \\ v_{x}-K \eta v_{z}=K \eta_{z} v & (x=0) .\end{cases}
$$

Let $S_{\delta}(v)=\int_{0}^{v} S_{\delta}^{\prime}(t) d t$ be a regularization of $v^{+}$with

$$
S_{\delta}^{\prime}(t)= \begin{cases}0 & \text { for } t \leq 0 \\ t / \delta & \text { for } 0 \leq t<\delta \\ 1 & \text { for } t \geq \delta\end{cases}
$$

Since $S_{\delta}^{\prime}(v) \in H^{1}\left(\mathbb{R}_{+}^{2}\right)$, we can multiply (2.6) by $S_{\delta}^{\prime}(v)$ and integrate by parts. It comes

$$
\begin{aligned}
\int_{\mathbf{R}_{+}^{2}}\left[\frac{1}{2} S_{\delta}(v)\right. & \left.+|\nabla v|^{2}\left(S_{\delta}^{\prime \prime}(v)\right)-\frac{1}{2}\left(v S_{\delta}^{\prime}(v)-S_{\delta}(v)\right)\right] d z d x \\
& =-K \int_{\mathbb{R}} \eta_{z}\left(v S_{\delta}^{\prime}(v)-S_{\delta}(v)\right) d z
\end{aligned}
$$


Remark that $\mid\left(v S_{\delta}^{\prime}(v)-S_{\delta}(v) \mid \leq \frac{1}{2} \min \left(v^{+}, \delta\right)\right.$, that $\forall t S_{\delta}^{\prime \prime}(t) \leq 0$ and that $v \in L^{1}\left(\mathbb{R}_{+}^{2}\right)$ (which is a consequence of $v \in L_{\rho}^{2}\left(\mathbb{R}_{+}^{2}\right)$ ). Then, since we assume here that $\eta_{z} \leq 0$, there holds

$$
\begin{aligned}
\frac{1}{2} \int_{\mathbb{R}_{+}^{2}} S_{\delta} v & \leq \frac{1}{4} \int_{\mathbb{R}_{+}^{2}} \min \left(v^{+}, \delta\right)-K \frac{\delta}{2} \int_{\mathbb{R}} \eta_{z} \\
& \leq \frac{1}{4} \int_{\mathbb{R}_{+}^{2}} \min \left(v^{+}, \delta\right)+K \frac{\delta}{2} .
\end{aligned}
$$

Let $\delta \rightarrow 0$. We deduce from dominated convergence that $\int_{\mathbb{R}_{+}^{2}} v^{+}=0$, which implies $v \leq 0$.

We now can prove the following existence theorem:

PROPOSITION 2.3. - The regularized problem $(2.1 \mathrm{H})$ admits a solution $u^{\varepsilon}$ in $C^{2, \alpha}\left(\overline{\mathbb{R}_{+}^{2}}\right)$ that verifies:

$$
\begin{gathered}
\left\|u^{\varepsilon}\right\|_{H_{\rho}^{1}\left(\mathbb{R}_{+}^{2}\right)} \leq C_{0} \quad \text { where } C_{0} \text { is independent of } \varepsilon \\
0 \leq u^{\varepsilon}(z, x) \leq \Lambda(z, x) \\
u^{\varepsilon}+\psi \text { is decreasing with respect to } z \\
u^{\varepsilon} \text { is decreasing with respect to } x
\end{gathered}
$$

Proof. - We shall apply the super-/sub-solution method - Sattinger [16]. Let the $C^{2, \alpha}$ sequence $u^{k}$ defined as follows. $u^{0} \equiv 0$ and, for $k \geq 0, u^{k+1}$ is the solution of

$$
\begin{cases}-\Delta u^{k+1}-\frac{1}{2}\left(z u_{z}^{k+1}+x u_{x}^{k+1}\right)=0 & \left(\mathbb{R}_{+}^{2}\right) \\ u_{x}^{k+1}-K \eta^{k}\left(u^{k+1}+\psi\right)_{z}=0 & (x=0)\end{cases}
$$

where $\eta^{k}=T^{\varepsilon} u^{k}+\psi$.

The definition of $T^{\varepsilon}$ as the solution of a well-chosen parabolic problem and the property $-\Lambda_{z z}-\frac{1}{2} z \Lambda_{z} \geq 0$ on $\{x=0\}$ imply that

$$
\forall \varepsilon>0 \quad T^{\varepsilon} \Lambda \leq \Lambda
$$

and that

$$
\left(\begin{array}{l}
0 \leq u^{k} \leq \Lambda \\
u^{k}+\psi \text { is decreasing along } z
\end{array}\right) \Longrightarrow\left(\begin{array}{l}
0 \leq \eta^{k} \leq \Lambda+\psi \\
\eta^{k} \text { is decreasing along } z
\end{array}\right) .
$$

Vol. $16, \mathrm{n}^{\circ} 2-1999$. 
So, recurrently, Lemma 2.2 shows that these properties are verified $\forall k \geq 0$. It also gives the property $\left\|u^{k}\right\|_{H_{\rho}^{1}\left(\mathbb{R}_{+}^{2}\right)} \leq C_{0}$.

We will now prove that the sequence $u^{k}$ is increasing. Let $w^{k+1}=$ $u^{k+1}-u^{k}$. We have $w^{1}=u^{1} \geq 0$. Since $T^{\varepsilon}$ is linear, this function $w^{k+1}$ verifies, for $k \geq 0$,

$$
\begin{cases}-\Delta w^{k+1}-\frac{1}{2}\left(z w_{z}^{k+1}+x w_{x}^{k+1}\right)=0 & \left(\mathbb{R}_{+}^{2}\right) \\ w_{x}^{k+1}=K \eta^{k-1} w_{z}^{k+1}+K T^{\varepsilon} w^{k}\left(u^{k+1}+\psi\right)_{z} & (x=0) .\end{cases}
$$

Suppose that $w^{k} \geq 0$. Then $K T^{\varepsilon} w^{k}\left(u^{k+1}+\psi\right)_{z} \leq 0$ and $w^{k+1}$ verifies

$$
w_{x}^{k+1}-K \eta^{k-1} w_{z}^{k+1} \leq 0 \quad(x=0) .
$$

Hence, we deduce from the maximum principle and Hopf's lemma that $w^{k+1} \geq 0$. Therefore, we prove recurrently that $u^{k} \leq u^{k+1}$ and obtain finally

$$
0 \leq u^{1} \leq u^{2} \leq \ldots \leq u^{k} \leq u^{k+1} \leq \ldots \leq \Lambda .
$$

Moreover, with $\left\|u^{k}\right\|_{H^{1}\left(\mathbb{A}_{+}^{2}\right)} \leq C$ and the definition of $T^{\varepsilon}$, if yields

$$
\left\|\eta^{k}\right\|_{C^{1, \alpha}(\mathbb{R})} \leq C_{\varepsilon} \text { and }\left\|u^{k}\right\|_{C^{2, \alpha}\left(\mathbb{R}_{+}^{2}\right)} \leq C_{\varepsilon}^{\prime}
$$

where $C_{\varepsilon}^{\prime}$ depends on $\varepsilon$ but not on $k$. The last estimate comes from the properties of the regular problem $(2.3 \mathrm{H})$.

Hence we can pass to the limit as $k \rightarrow+\infty$ and get $u^{\varepsilon} \in C^{2, \alpha^{\prime}}\left(\overline{\mathbb{R}_{+}^{2}}\right)$ solution of $(2.1 \mathrm{H})$. We also pass to the limit for the properties $(2.8)$ and (2.9) and apply Lemma 2.2 once again, with $\eta=T^{\varepsilon} u^{\varepsilon}+\psi$ to obtain (2.7).

To prove (2.10), set $v^{\varepsilon}=u_{x}^{\varepsilon}$. This function verifies

$$
\begin{cases}-\Delta v^{\varepsilon}-\frac{1}{2}\left(z v_{z}^{\varepsilon}+x v_{x}^{\varepsilon}\right)-\frac{1}{2} v^{\varepsilon}=0 & \left(\mathbb{R}_{+}^{2}\right) \\ v^{\varepsilon}=K\left(u^{\varepsilon}+\psi\right)\left(u^{\varepsilon}+\psi\right)_{z} \leq 0 & (x=0) .\end{cases}
$$

Multiply the first equation by $\left(v^{\varepsilon}\right)^{+}$and integrate; with $\left(v^{\varepsilon}\right)_{(x=0)}^{+}=0$ it comes $\left(v^{e}\right)^{+} \equiv 0$ on $\overline{\mathbb{R}_{+}^{2}}$. So $u^{e}$ is decreasing with respect to $x$.

\subsection{Further estimates}

The crucial point that will allow us to pass to the limit is the following estimate.

Proposition 2.4. - Let $u^{\varepsilon}$ the solution of the regularized problem $(2.1 \mathrm{H})$. There exists a constant $C$ independent of $\varepsilon$ such that

$$
\left\|u^{\varepsilon}\right\|_{H^{3 / 2}\left(\mathbb{R}_{+}^{2}\right)} \leq C \text {. }
$$


Proof. - We proceed in two steps: first we estimate $\left\|\gamma u_{z}^{\varepsilon}\right\|_{\left.\left.L^{2}(]-\infty, a\right]\right)}$ for $a \in \mathbb{R}$, then we extend to $\left\|u^{\varepsilon}\right\|_{H^{3 / 2}\left(\mathbb{R}_{+}^{2}\right)}$.

Step 1. $-u^{\varepsilon} \in H^{2}\left(\mathbb{R}_{+}^{2}\right)$ so we can take $u_{z}^{\varepsilon}$ as a test function in the variational formulation associated to $(2.1 \mathrm{H})$ :

$$
\int_{\mathbf{R}_{+}^{2}} \nabla u^{\varepsilon} \nabla u_{z}^{\varepsilon}-\frac{1}{2} \int_{\mathbf{R}_{+}^{2}}\left(z u_{z}^{\varepsilon}+x u_{x}^{\varepsilon}\right) u_{z}^{\varepsilon}=-K \int_{\mathbf{R}^{\prime}}\left(T^{\varepsilon} u^{\varepsilon}+\psi\right)\left(u_{z}^{\varepsilon}+\psi_{z}\right) u_{z}^{\varepsilon}
$$

Apply (2.7), (2.8), (2.9) and $\int_{\mathbb{R}_{+}^{2}} \nabla u^{\varepsilon} \nabla u_{z}^{\varepsilon}=0$, it follows

$$
K \int_{\mathbb{R}}\left(T^{\varepsilon} u^{\varepsilon}+\psi\right) u_{z}^{\varepsilon 2} \leq C
$$

where $C$ is independent of $\varepsilon$.

Since $\psi \leq\left(T^{\varepsilon} u^{\varepsilon}+\psi\right)$ and $\psi$ decreases, we obtain

$$
\forall a \in \mathbb{R} \quad\left\|\gamma u_{z}^{\varepsilon}\right\|_{L^{2}(]-\infty, a[)} \leq \frac{C}{\psi(a)}
$$

Step 2. - In this part we estimate $u_{z}^{\varepsilon}$ on $[a,+\infty[$ thanks to (2.12).

LEMMA 2.5. - Let $g \in L^{2}(\mathbb{R})$ and $u \in H^{3 / 2}\left(\mathbb{R}_{+}^{2}\right) \cup H_{\rho}^{1}\left(\mathbb{R}_{+}^{2}\right)$ verifying

$$
\begin{cases}-\Delta u-\frac{1}{2}\left(z u_{z}+x u_{x}\right)=0 & \left(\mathbb{R}_{+}^{2}\right) \\ u_{x}=g & (x=0)\end{cases}
$$

Then $\|u\|_{H^{3 / 2}\left(\mathbb{R}_{+}^{2}\right)} \leq C\|g\|_{L^{2}(\mathbb{R})}$.

The term $-\frac{1}{2}\left(z u_{z}+x u_{x}\right)$ is indeed treated as a $L^{2}$ right-hand side. From this lemma, one deduces

LEMMA 2.6. - Let $a>0$ and $u \in H^{3 / 2}\left(\mathbb{R}_{+}^{2}\right)$ verifying $-\Delta u-\frac{1}{2}\left(z u_{z}+\right.$ $\left.x u_{x}\right)=0$. Then there exists a constant $C$ such that

(2.13) $\|u\|_{H^{3 / 2}(] a,+\infty\left[\times \mathbb{R}_{+}\right)} \leq C\left(\|u\|_{L^{2}(] a-1,+\infty\left[\times \mathbb{R}_{+}\right)}+\left\|\gamma u_{x}\right\|_{L^{2}(] a-1,+\infty[)}\right)$.

End of the proof of Proposition 2.4. - We have seen that $T^{\varepsilon} u^{\varepsilon} \leq \Lambda$, so with the decrease of $\Lambda$ and $\psi$ at $z \rightarrow+\infty$, there exists a constant $C$ such that

$$
\text { for } z \geq a-1, \quad K\left(T^{\varepsilon} u^{\varepsilon}+\psi\right) \leq C e^{-a^{2} / 8}
$$

and

$$
\left\|u^{\varepsilon}\right\|_{L^{2}(] a-1,+\infty[) \times \mathbf{R}_{+}} \leq C e^{-a^{2} / 8}
$$

Vol. 16, $n^{\circ} 2-1999$ 
Thus, if we replace $\gamma u_{x}^{\varepsilon}$ by $K\left(I^{\varepsilon} u^{\varepsilon}+\psi\right)\left(u_{z}^{\varepsilon}+\psi_{z}\right)$ in (2.13), it follows

$$
\left\|u^{\varepsilon}\right\|_{H^{3 / 2}(] a_{2}+\infty\left[\times \mathbb{R}_{1}\right)} \leq\left(C_{1}+C_{2}\left\|\gamma u_{z}^{\varepsilon}\right\|_{L^{2}(j a-1,+\infty[)}\right) e^{-a^{2} / 8} .
$$

Then we use a trace theorem: $\left\|\gamma u_{z}^{\varepsilon}\right\|_{L^{2}(] a,+\infty[)} \leq C_{3}\left\|u^{\varepsilon}\right\|_{H^{3 / 2}(] a,+\infty\left[\times \mathbb{R}_{+}\right)}$. This works because $\partial / \partial z$ is a tangential derivative.

We choose $a>0$ such that $C_{2} C_{3} e^{-a^{2} / 8} \leq 1 / 2$; it gives

$$
\left\|u^{\varepsilon}\right\|_{H^{3 / 2}(] a,+\infty\left[\times \mathbb{F}_{+}\right)} \leq C\left(1+\left\|\gamma u^{\epsilon}\right\|_{L^{2}(] a-1, a[}\right) .
$$

With the result of Step 1 we can conclude the proof.

\subsection{Passing to the limit}

Let $\varepsilon \rightarrow 0$ and take (2.7), (2.8), (2.11); we can extract from $u^{\varepsilon}$ a subsequence that converges to $u$ in $H^{3 / 2}\left(\mathbb{R}_{+}^{2}\right)$ weak, $L^{2}\left(\mathbb{R}_{+}^{2}\right)$ strong and $L^{\infty}\left(\mathbb{R}_{+}^{2}\right)$ weak *. Indced, $H_{\rho}^{1}\left(\mathbb{R}_{+}^{2}\right)$ is compact in $L^{2}\left(\mathbb{R}_{+}^{2}\right)$.

Then let $\varphi \in C_{c}^{\infty}\left(\overline{\mathbb{R}_{+}^{2}}\right)$. We know that $\left\|\gamma u^{\varepsilon}\right\|_{H^{1}(\mathbb{R})} \leq C$. Thus if $\Xi \subset \mathcal{O} \subset \mathbb{R}$ is the compact support of $\gamma \varphi$ and $\mathcal{O}$ is bounded, then after another extraction, $\gamma u^{\varepsilon}$ converges to $\gamma u$ in $L^{2}(\mathcal{O})$ strong. Write the variational formulation of $(2.1 \mathrm{H})$ applied to the test-function $\varphi$ and let $\varepsilon \rightarrow 0$.

The crucial term is the nonlinear boundary term, that we write

$$
\int_{\mathbb{R}}\left(T^{\varepsilon} u^{\varepsilon} u_{z}^{\varepsilon}-u u_{z}\right) \varphi=\int_{\mathbb{B}}\left(T^{\varepsilon} u^{\varepsilon}-u^{\varepsilon}\right) u_{z}^{\varepsilon} \varphi+\int_{\mathbb{R}}\left(u^{\varepsilon}-u\right) u_{z}^{\varepsilon} \varphi+\int_{\mathbb{R}} u\left(u_{z}^{\varepsilon}-u_{z}\right) \varphi .
$$

These three integrals converge to zero:

- For the first one we use the fact that $T^{\varepsilon} \rightarrow$ Id uniformly on every bounded subset of $H^{1 / 2}(\mathbb{R})$ and that $\gamma u_{z}^{\varepsilon} \varphi$ is bounded in $H^{-1 / 2}(\mathbb{R})$.

- For the second one, $\gamma u^{\varepsilon} \rightarrow \gamma u$ in $L^{2}(\mathcal{O})$ strong and $\gamma u_{z}^{\varepsilon} \varphi$ is bounded in $L^{2}(\Xi)$.

- The third one converges to zero since $\gamma u_{z}^{\varepsilon} \rightarrow \gamma u_{z}$ in $L^{2}(\mathbb{R})$ weak.

We have proved then that

$$
\begin{aligned}
& \forall \varphi \in C_{c}^{\infty}\left(\overline{\mathbb{R}_{+}^{2}}\right) \\
& \int_{\mathbf{R}_{+}^{2}} \nabla u \nabla \varphi-\frac{1}{2} \int_{\mathbf{R}_{+}^{2}}\left(z u_{z}+x u_{x}\right) \varphi=-K \int_{\mathbf{R}}(u+\psi)(u+\psi)_{z} \varphi .
\end{aligned}
$$

The right-hand side makes sense since $\gamma(u+\psi) \varphi \in H^{1 / 2}(\mathbb{R})$ and $\gamma(u+\psi)_{z} \in H^{-1 / 2}(\mathbb{R})$ but we can also notice that

$$
\frac{1}{2} \gamma\left[(u+\psi)^{2}\right]_{z} \in H^{-1 / 2}(\mathbb{R})
$$


so we can extend by density to $\varphi \in H^{1}\left(\mathbb{R}_{+}^{2}\right)$, writing

$\forall \varphi \in H^{1}\left(\mathbb{R}_{+}^{2}\right)$

$\int_{\mathbb{R}_{\mid}^{2}} \nabla u \nabla \varphi-\frac{1}{2} \int_{\mathbb{R}_{1}^{2}}\left(z u_{z}+x u_{x}\right) \varphi=-K\left\langle\frac{1}{2}\left[(u+\psi)^{2}\right]_{z}, \varphi\right\rangle_{H^{-1 / 2}(\mathbb{P}), H^{1 / 2}(\mathbb{P})}$.

Thus $u$ is a weak solution of $(1.4 \mathrm{H})$. We can also pass to the limit in (2.9), (2.10), (2.8) and take Lemma 2.1 with $\sigma=2$ to prove the properties stated in Theorem 1.1.

At the stage, we only have a weak $H^{3 / 2}$ solution. Nevertheless, this regularity is enough to prove the uniqueness of the solution in this class, as we show in the next subsection.

Remark. - These estimates would be valid for positive solutions of more general elliptic equations, for example with suitable polynomial coefficients and suitable nonlinear dependence in $u$ and $\nabla u$.

\subsection{Uniqueness}

Let $u_{1}$ and $u_{2}$ be two solutions of $(1.4 \mathrm{H})$ in $H^{3 / 2}\left(\mathbb{R}_{+}^{2}\right)$. We shall prove that $u_{1}=u_{2}$. Remark that we only suppose the $H^{3 / 2}$ regularity of these solutions; we do not know a priori that they are bounded by $\Lambda$ or that $u_{1}+\psi$ or $u_{2}+\psi$ is decreasing with respect to $z$.

Set

$$
R_{\delta}(w)= \begin{cases}\frac{w^{\delta+1}}{(1+\delta) \delta^{\delta}} & \text { for } 0 \leq w \leq \delta \\ w-\frac{\delta^{2}}{1+\delta} & \text { for } w \geq \delta\end{cases}
$$

There holds

$\int_{\mathbf{R}_{+}^{2}} R_{\delta}^{\prime \prime}(w)|\nabla w|^{2}+\int_{\mathbf{R}_{+}^{2}} R_{\delta}(w)+\frac{K}{2} \int_{\mathbf{R}}\left(u_{1}+\psi+u_{2}+\psi\right)_{z}\left(w R_{\delta}^{\prime}(w)-R_{\delta}(w)\right)=0$.

$\gamma\left(u_{1}+\psi+u_{2}+\psi\right)_{z} \in L^{2}(\mathbb{R})$ and $\gamma\left(w R_{\delta}^{\prime}(w)-R_{\delta}(w)\right) \in L^{2}(\mathbb{R})$ so we can apply Schwartz's formula to the boundary term, denoted B.T.:

$$
|B . T .| \leq \frac{K}{2}\left(\int_{\mathbb{R}}\left(u_{1}+\psi+u_{2}+\psi\right)_{z}^{2} \cdot \int_{\mathbb{R}}\left(w R_{\delta}^{\prime}(w)-R_{\delta}(w)\right)^{2}\right)^{1 / 2} .
$$

We remark that

$$
\forall t \quad 0 \leq t R_{\delta}^{\prime}(t)-R_{\delta}(t) \leq \min \left(\frac{\delta^{2}}{(1+\delta)}, \frac{t^{1+\delta}}{(1+\delta) \delta^{\delta-1}}\right)
$$


and, to control $\int_{\mathbb{B}}\left(w R_{\delta}^{\prime}(w)-R_{\delta}(w)\right)^{2}$, we use a balance between the two terms of the min function: let $(.)^{2}=(.)^{2(1-\alpha)}(.)^{2 \alpha}$ with $\alpha=1 /(1+\delta)$. It gives

$$
\begin{aligned}
\int_{\mathbb{R}}\left(w R_{\delta}^{\prime}(w)-R_{\delta}(w)\right)^{2} & \leq\left(\frac{\delta^{2}}{(1+\delta)}\right)^{\frac{2 \delta}{1+\delta}} \int_{\mathbb{R}}\left(\frac{w^{1+\delta}}{(1+\delta) \delta^{\delta-1}}\right)^{\frac{2}{1+\delta}} \\
& \leq \frac{\delta^{2}}{(1+\delta)^{2}} \int_{\mathbb{R}} w^{2}
\end{aligned}
$$

Hence

$$
|B . T .| \leq C \delta\left\|\left(u_{1}+u_{2}+2 \psi\right)_{z}\right\|_{H^{1 / 2}\left(\mathbf{R}_{+}^{2}\right)}\|w\|_{H^{1 / 2}\left(\mathbf{R}_{+}^{2}\right)} .
$$

Since $R_{\delta}^{\prime \prime}(w) \geq 0$, if we fix $w$, then $0 \leq \int_{\mathbb{R}_{+}^{2}} R_{\delta}(w) \leq C \delta$.

The proof is terminated by an application of the Beppo Levi Theorem.

Remark. - It would be possible at this stage to prove the uniqueness of non-increasing $H_{l o c}^{\perp}$ solutions. Here it is only of moderate interest, but it will turn out to be important in Part 2 of our study.

\section{SMOOTHNESS OF THE SELF-SIMILAR SOLUTION}

\section{1. $H^{2}\left(\mathbb{R}_{+}^{2}\right)$ estimate}

Lemma 3.1. - Let $u$ be the solution of (1.4). There exists $C>0$ such that $\left\|\gamma u_{z}\right\|_{L^{3}(\mathbf{R})}<C$.

Proof. - Remark first that in this lemma we deal with $\gamma u_{z}=\gamma U_{z}-\psi_{z}$ so since $\psi_{z}=\frac{1}{2 \sqrt{\pi}} \operatorname{cxp}\left(\frac{z^{2}}{4}\right)$, it is equivalent to estimate $\left\|\gamma U_{z}\right\|_{L^{3}}$. Then it will be useful to recall that we control the sign of $U_{z}$ (and of $U_{z}^{\varepsilon}$ ), which is always negative.

We now come back to the regularized problem $(2.1 \mathrm{H}): u^{\varepsilon}$ is a solution of this system, and $U^{\varepsilon}=u^{\varepsilon}+\psi$ is the solution of the non-homogeneous associated problem $(2.1 \mathrm{NH})$. Here $C$ will denote a constant independent of $\varepsilon$. With the Sobolev embeddings and $\left\|u^{\varepsilon}\right\|_{H^{3 / 2}\left(\mathbb{R}_{+}^{2}\right)} \leq C$, the two functions $u_{z}^{\varepsilon}$ and $u_{x}^{\varepsilon}$ are bounded in $L^{3}\left(\mathbb{R}_{+}^{2}\right)$ and in $L^{4}\left(\mathbb{R}_{+}^{2}\right)$, independently of $\varepsilon$. Hence, we write, if $\chi$ is a bounded function,

$$
\left|\frac{1}{2} \int_{\mathbb{R}_{+}^{2}} \chi \partial_{\beta_{1}} u^{\varepsilon} \partial_{\beta_{2}} u^{\varepsilon} \partial_{\beta_{3}} u^{\varepsilon}\right| \leq C,
$$


where $\partial_{\beta_{i}} u^{\varepsilon}$ denotes any first derivative of $u^{\varepsilon}$. Moreover, since $\nabla u^{\varepsilon}$ is bounded in $L_{\rho}^{2}\left(\mathbb{R}_{+}^{2}\right)$, there holds

$$
\left|\frac{1}{2} \int_{\mathbb{R}_{1}^{2}} \chi\left(z u_{z}^{\varepsilon}+x u_{x}^{\varepsilon}\right) \partial_{\beta_{1}} u^{\varepsilon} \partial_{\beta_{2}} u^{\varepsilon}\right| \leq C .
$$

Since $u^{\varepsilon} \in H^{2}\left(\mathbb{R}_{+}^{2}\right) \cap C^{2, \alpha}\left(\overline{\mathbb{R}_{+}^{2}}\right)$, the functions $\partial_{\beta_{1}} u^{\varepsilon} \partial_{\beta_{2}} u^{\varepsilon}$ are in $H^{1}\left(\mathbb{R}_{+}^{2}\right)$, and can be taken as test-functions in the variational formulation of $(2.1 \mathrm{H})$.

Let now $v(z, x)$ be a smooth function such that $v$ and its derivatives are small enough at the infinity, and $\chi(z)$ be a smooth function in $W^{1, \infty}(\mathbb{R})$ ( $\chi$ is a cut-off function and shall be precised later). After developments and integrations by parts, we obtain the two equalities:

$$
\begin{aligned}
\int_{\mathbb{R}_{+}^{2}}(-\Delta v) v_{z} v_{x} \chi & =\int_{\mathbb{R}}\left[\frac{1}{2}\left(v_{x}\right)^{2} v_{z}-\frac{1}{6}\left(v_{z}\right)^{3}\right] \chi \\
& +\int_{\mathbb{R}_{+}^{2}}\left[\frac{1}{2}\left(v_{z}\right)^{2} v_{x}-\frac{1}{6}\left(v_{x}\right)^{3}\right] \chi^{\prime},
\end{aligned}
$$

and

$$
\begin{aligned}
\int_{\mathbb{R}_{+}^{2}}(-\Delta v)\left[\left(v_{x}\right)^{2}-\left(v_{z}\right)^{2}\right] \chi & =\int_{\mathbb{R}}\left[\frac{1}{3}\left(v_{x}\right)^{3}-v_{x}\left(v_{z}\right)^{2}\right] \chi \\
& +\int_{\mathbb{R}_{+}^{2}}\left[\left(v_{x}\right)^{2} v_{z}+\frac{1}{3}\left(v_{z}\right)^{3}\right] \chi^{\prime}
\end{aligned}
$$

We multiply the equation verified by $u^{\varepsilon}(2.1 \mathrm{H})$ inside $\mathbb{R}_{+}^{2}$ by the testfunctions $u_{z}^{\varepsilon} u_{x}^{\varepsilon} \chi$ or $\left[\left(u_{x}^{\varepsilon}\right)^{2}-\left(u_{z}^{\varepsilon}\right)^{2}\right] \chi$, then integrate it. Recall that on the boundary there holds $u_{x}^{\varepsilon}=K T^{\varepsilon}\left(U^{\varepsilon}\right) U_{z}^{\varepsilon}$. By (3.2), (3.3), (3.4), (3.1), and since $\gamma U_{z}^{\varepsilon}$ and $\psi_{z}$ are bounded in $L^{2}(\mathbb{R})$, it comes

$$
\int_{\mathbb{R}} \chi \cdot\left(\left(K T^{\varepsilon} U^{\varepsilon}\right)^{2}-\frac{1}{3}\right)\left(U_{z}^{\varepsilon}\right)^{3} d z=\mathcal{O}(1),
$$

and

$$
\int_{\mathbf{R}} \chi \cdot\left(\frac{\left(K T^{\varepsilon} U^{\varepsilon}\right)^{3}}{3}-\left(K T^{\varepsilon} U^{\varepsilon}\right)\right)\left(U_{z}^{\varepsilon}\right)^{3} d z=\mathcal{O}(1)
$$

If $K<\frac{1}{\sqrt{3}}$ then (3.5) implies directly the estimate, with $\chi \equiv 1$, since $0 \leq U^{\varepsilon} \leq 1$.

Vol. 16, $n^{\circ}$ 2-1999. 
Otherwise, we know - now for a long time - that $U^{\varepsilon}$ is continuous, with uniformly bounded moduli of continuity. For $\delta$ small enough, one can find $Z_{\delta}^{\prime}<Z_{\delta}$ and $\varepsilon_{\delta}$ such that for $\varepsilon<\varepsilon_{\delta}, z \geq Z_{\delta}$, there holds $K T^{\varepsilon} U^{\varepsilon} \leq \frac{1}{\sqrt{3}}-\delta$ and for $\varepsilon<\varepsilon_{\delta}, z \leq Z_{\delta}^{\prime}$, there holds $K T^{\varepsilon} U^{\varepsilon} \geq \frac{1}{\sqrt{3}}-\delta / 2$. Then we take the nonnegative truncation function $\chi_{1}(z) \in C^{\infty}(\mathbb{R})$ such that

$$
\begin{cases}\chi_{1}(z)=1 & \text { if } z \geq Z_{\delta} \\ \chi_{1}(z)=0 & \text { if } z \leq Z_{\delta}^{\prime} .\end{cases}
$$

By (3.5) we have $\int_{\mathbb{R}}\left(U_{z}^{\varepsilon}\right)^{3} \chi_{1} \leq C_{\delta}$, for $\varepsilon<\varepsilon_{\delta}$, thus

$$
\left\|U_{z}^{\varepsilon}\right\|_{\left.L^{3}(\} Z_{\delta},+\infty\right)} \leq C_{\delta}^{\prime}
$$

If $K>\frac{1}{\sqrt{3}}$, we obtain, with a similar argument, a point $Y_{\delta}$ such that, for $z \leq Y_{\delta}$ and $\varepsilon<\varepsilon_{\delta}$, there holds $K T^{\varepsilon} U^{\varepsilon} \geq \frac{1}{\sqrt{3}}+\delta$ and

$$
\left\|U_{z}^{\varepsilon}\right\|_{L^{3}(]-\infty, Y_{\delta}[)} \leq C_{\delta} .
$$

On $\left[Y_{\delta}, Z_{\delta}\right],(3.6)$ enables to conclude. Indeed $\frac{1}{\sqrt{3}}$ is not a zero of $\frac{X^{3}}{3}-X$, so with a truncation function $\chi_{2}$ that takes the value 1 on $\left[Y_{\delta}, Z_{\delta}\right]$ and vanishes on $\mathbb{R} \backslash\left[Y_{2 \delta}, Z_{2 \delta}\right]$, and $\delta$ small enough, we get, for $z \in\left[Y_{\delta}, Z_{\delta}\right]$,

$$
\frac{\left(K T^{\varepsilon} U^{\varepsilon}\right)^{3}}{3}-\left(K T^{\varepsilon} U^{\varepsilon}\right)<-\frac{8}{9 \sqrt{3}}+\delta^{\prime},
$$

where $\delta^{\prime}$ is a small real number. Hence, by (3.6):

$$
\left\|U_{z}^{\varepsilon}\right\|_{L^{3}(] Y_{\delta}, Z_{\delta}[)} \leq C_{\delta}
$$

If $K=\frac{1}{\sqrt{3}}$, the argument is the same, with $Y_{\delta}$ replaced by $-\infty$.

To prove the $H^{2}$ regularity of the self-similar solution, we will use the Nirenberg translations method. For that, we introduce some notations and a technical lemma.

$$
\begin{array}{cl}
\text { for } h \in \mathbb{R}^{*} & \tau_{h} u(z, x)=u(z+h, x) \\
& D_{h} u=\frac{\tau_{h} u-u}{|h|} .
\end{array}
$$


LEMMA 3.2. $-D_{h}$ possesses the following properties:

(i) $D_{h}$ is linear and $\forall s, p W^{s, p}\left(\mathbb{R}_{+}^{2}\right)$ is stable by $D_{h}$;

(ii) $D_{h}$ and $\nabla$. commute;

(iii) $\forall u, v D_{h}\left(u^{2}\right)=2 u D_{h} u+|h|\left(D_{h} u\right)^{2}$

$D_{h}(z u)=z D_{h} u+\frac{h}{|h|} \tau_{h} u$

$D_{h}(u v)=v D_{h} u+\left(D_{h} v\right)\left(\tau_{h} u\right)$;

(iv) $\forall(u, v) \in\left(L^{2}\left(\mathbb{R}_{+}^{2}\right)\right)^{2} \int_{\mathbb{R}_{+}^{2}} u . D_{-h} v=\int_{\mathbb{R}_{+}^{2}} D_{h} u . v$;

(v) $\forall u \in H^{1}\left(\mathbb{R}_{+}^{2}\right)\left\|D_{h} u\right\|_{L^{2}\left(\mathbb{R}_{+}^{2}\right)} \leq\|\nabla u\|_{\left(L^{2}\left(\mathbb{R}_{+}^{2}\right)\right)^{2}}$;

(vi) $\forall v \in W^{1,3}(\mathbb{R})\left|\int_{\mathbb{R}} v_{z}\left(D_{h} v\right)^{2}\right| \leq\left\|v_{z}\right\|_{L^{3}(\mathbb{R})}^{3}$.

Proof. - (i), (ii) and (iii) are immediate and come from the expression of $D_{h}$ after straightforward calculations.

(iv) is also obvious after an integration by parts.

$(v)$ is proved in [5].

(vi) can be proved as $(v)$ : first for $v \in C^{1}(\mathbb{R})$, after an integration, we have

$$
D_{h} v=\frac{h}{|h|} \int_{0}^{1} v^{\prime}(z+t h) d t
$$

then

$$
\begin{aligned}
\left|\int_{\mathbb{R}} v_{z}\left(D_{h} v\right)^{2} d z\right| & \leq \int_{0}^{1}\left(\int_{\mathbb{R}}\left|v_{z}\right| \cdot\left(v_{z}(z+t h)\right)^{2} d z\right) d t \\
& \leq\left\|v_{z}\right\|_{L^{3}(\mathbb{R})}\left(\int_{\mathbb{R}}\left(v_{z}(z+t h)\right)^{3} d z\right)^{2 / 3} \\
& \leq\left\|v_{z}\right\|_{L^{3}(\mathbb{R})}^{3} .
\end{aligned}
$$

We conclude by a density argument.

Proposition 3.3. - Let $u$ be the solution of $(1.4 \mathrm{H})$. There exists $C>0$ such that $\|u\|_{H^{2}\left(\mathbb{R}_{+}^{2}\right)}<C$.

Proof. - We first make the following remark.

Suppose that $\left\|u_{z}\right\|_{H^{1}\left(\mathbb{R}_{+}^{2}\right)}<C_{1}$; then $\gamma u$ is bounded in $H^{3 / 2}(\mathbb{R})$. Thus, since $u$ verifies $-\Delta u-\frac{1}{2}\left(z u_{z}+x u_{x}\right)=0$ in $\left(\mathbb{R}_{+}^{2}\right)$, the estimate in $H^{2}\left(\mathbb{R}_{+}^{2}\right)$ follows - Agmon-Douglis-Nirenberg [1].

Therefore the point is to estimate $u_{z}$ in $H^{1}\left(\mathbb{R}_{+}^{2}\right)$. As in Lemma 3.1, $U_{z}=u_{z}+\psi_{z}$, where $U$ is the solution of the non homogeneous problem Vol. 16, $n^{\circ} 2-1999$. 
(1.4NH). We have

$$
\begin{aligned}
& \forall \varphi \in H^{1}\left(\mathbb{R}_{+}^{2}\right) \\
& \int_{\mathbb{R}_{+}^{2}} \nabla u \nabla \varphi-\frac{1}{2} \int_{\mathbb{R}_{+}^{2}}\left(z u_{z}+x u_{x}\right) \varphi+\frac{K}{2} \int_{\mathbb{R}^{\prime}}\left(U^{2}\right)_{z} \varphi=0 .
\end{aligned}
$$

The idea of the proof is to take $\varphi=u_{z z}$ in (3.7), but we only know that $u_{z z} \in H^{-1 / 2}\left(\mathbb{R}_{+}^{2}\right)$ : it is not an acceptable test function. To avoid this difficulty, we use the Nirenberg translations method.

With Lemma 3.2 (i)

$$
\varphi=D_{-h} D_{h} u \in H^{1}\left(\mathbb{R}_{+}^{2}\right) .
$$

Then, with the properties $(i),(i i),(i i i)$ of this lemma, we calculate and estimate the different terms of (3.7):

$$
\begin{aligned}
& \int_{\mathbb{R}_{+}^{2}} \nabla u \nabla \varphi=\int_{\mathbb{R}_{+}^{2}}\left(D_{h}(\nabla u)\right)^{2} \\
& \left|-\frac{1}{2} \int_{\mathbb{R}_{+}^{2}} x u_{x} \varphi\right|=\frac{1}{4} \int_{\mathbb{R}_{+}^{2}}\left(D_{h} u\right)^{2} \leq \frac{1}{4}\|\nabla u\|_{\left(L^{2}\left(\mathbb{R}_{+}^{2}\right)\right)^{2}}^{2} \text { with }(v) ; \\
& \left|-\frac{1}{2} \int_{\mathbb{R}_{+}^{2}} z u_{z} \varphi\right|=\left|\frac{1}{4} \int_{\mathbb{R}_{+}^{2}}\left(D_{h} u\right)^{2}+\frac{h}{2|h|} \int_{\mathbb{R}_{+}^{2}} u_{z} D_{-h} u\right| \\
& \leq \frac{1}{4}\|\nabla u\|_{\left(L^{2}\left(\mathbb{R}_{+}^{2}\right)\right)^{2}}^{2}+\frac{1}{2}\left\|u_{z}\right\|_{L^{2}\left(\mathbb{R}_{+}^{2}\right)}\left\|D_{-h} u\right\|_{L^{2}\left(\mathbb{R}_{+}^{2}\right)} \\
& \text { with }(v) \text { and Cauchy-Schwarz formula } \\
& \leq \frac{3}{4}\|\nabla u\|_{\left(L^{2}\left(\mathrm{R}_{+}^{2}\right)\right)^{2}}^{2} \\
& \frac{K}{2} \int_{\mathbb{R}}\left(U^{2}\right)_{z} \varphi=\frac{K}{2} \int_{\mathbb{R}}\left(U^{2}\right)_{z} D_{-h} D_{h} U-\frac{K}{2} \int_{\mathbb{R}}\left(U^{2}\right)_{z} D_{-h} D_{h} \psi \\
& =-\frac{K}{2} \int_{\mathbb{R}} 2 U\left(D_{h} U\right)\left(D_{h} U\right)_{z}-\frac{K}{2}|h| \int_{\mathbb{R}}\left(D_{h} U\right)^{2}\left(D_{h} U\right)_{z} \\
& +\frac{K}{2} \int_{\mathbb{R}}\left(U^{2}\right) D_{-h} D_{h} \psi_{z} \\
& =\frac{K}{2} \int_{\mathbb{R}} U_{z}\left(D_{h} U\right)^{2}+\frac{K}{2} \int_{\mathbb{R}}\left(U^{2}\right) D_{-h} D_{h} \psi_{z} \\
& \left|\frac{K}{2} \int_{\mathbb{R}}\left(U^{2}\right)_{z} \varphi\right| \leq{ }_{2}^{K}\left\|\gamma U_{z}\right\|_{L^{3}(\mathrm{H})}^{3}+C\|\gamma U\|_{L^{2}(\mathfrak{R})}^{2} \\
& \text { with Lemmas 3.1, } 3.2 \text { (vi). }
\end{aligned}
$$


Finally

$$
\int_{\mathbb{R}_{+}^{2}}\left(D_{h}(\nabla u)\right)^{2} \leq\|\nabla u\|_{\left(L^{2}\left(\mathbb{R}_{+}^{2}\right)\right)^{2}}^{2}+\frac{K}{2}\left\|\gamma U_{z}\right\|_{L^{3}(\mathbb{R})}^{3}+C\|\gamma U\|_{L^{2}(\mathbb{R})}^{2}
$$

and the right-hand side is uniformly bounded in $\varepsilon$. As in [5], we can deduce from this inequality that $\left\|\nabla u_{z}\right\|_{\left(L^{2}\left(\mathbb{R}_{+}^{2}\right)\right)^{2}} \leq C$. We conclude as explained at the beginning of the proof.

\section{2. $C^{\infty}$ smoothness}

Two lemmas will lead to the conclusion.

LEMMA 3.4. - Let $u$ be the solution of $(1.4 \mathrm{H})$. If $\gamma u_{z} \in L^{\infty}(\mathbb{R})$ then $u \in C^{1, \alpha}\left(\overline{\mathbb{R}_{+}^{2}}\right)$.

Proof. - We shall prove that $u \in H^{3}\left(\mathbb{R}_{+}^{2}\right)$ which implies the result with the Sobolev embeddings. With Proposition 3.3 we already have $u \in H^{2}\left(\mathbb{R}_{+}^{2}\right)$. Let $v=-u_{z}$ and $V=-U_{z}=v-\psi_{z}$; the assumption of the lemma is $L=\|\gamma v\|_{\infty(\mathbb{R})}<+\infty$. Moreover, this function $v$ is solution of the system

$$
\begin{cases}-\Delta v-\frac{1}{2}\left(z v_{z}+x v_{x}\right)-\frac{1}{2} v=0 & \left(\mathbb{R}_{+}^{2}\right) \\ v_{x}=K U V_{z}-K V^{2} & (x=0)\end{cases}
$$

Thus

$\forall \varphi \in H^{1}\left(\mathbb{R}_{+}^{2}\right)$

$\int_{\mathbb{R}_{+}^{2}} \nabla v \nabla \varphi-\frac{1}{2} \int_{\mathbb{R}_{+}^{2}}\left(z v_{z}+x v_{x}\right) \varphi-\frac{1}{2} \int_{\mathbb{R}_{+}^{2}} v \varphi+K \int_{\mathbb{R}}\left(U V_{z}-V^{2}\right) \varphi=0$.

Remark that all the terms are well defined in this equation.

As in the last section, we use the Nircnberg translations and set $\varphi=D_{-h} D_{h} v \in H^{1}\left(\mathbb{R}_{+}^{2}\right)$. A calculation similar as before, with the technical Lemma 3.2, gives

$$
\int_{\mathbb{R}_{+}^{2}} \nabla v \nabla \varphi=\int_{\mathbb{R}_{+}^{2}}\left(D_{h}(\nabla v)\right)^{2}
$$

and

$$
\left|-\frac{1}{2} \int_{\mathbb{R}_{+}^{2}}\left(z v_{z}+x v_{x}+v\right) \varphi\right| \leq \frac{1}{2}\|\nabla v\|_{\left(L^{2}\left(\mathbb{R}_{+}^{2}\right)\right)^{2}}^{2}
$$


The new term is the boundary term

$$
K \int_{\mathbb{R}}\left(U V_{z}-V^{2}\right) \varphi=\cdots=-K \underbrace{\int_{\mathbb{R}} v D_{h} u D_{h} v_{z}}_{I_{1}}-\frac{K}{2} \underbrace{\int_{\mathbb{R}} \tau_{h} v\left(D_{h} v\right)^{2}}_{I_{2}}+I_{3},
$$

where $I_{3}$ is a lower order term in $u, v$, also involving $\psi$ and its derivative; this term is easily estimated.

We can estimate the two integrals $I_{1}$ and $I_{2}$ with the assumption on $L$ :

$$
\begin{aligned}
\left|I_{2}\right| & \leq L \int_{\mathbb{R}}\left(D_{h} v\right)^{2} \\
& \leq C L\left\|D_{h} v\right\|_{H^{1 / 2}\left(\mathbb{R}_{+}^{2}\right) \quad \text { with a trace theorem }}^{2} \\
& \leq \frac{C L}{2 A}\left\|D_{h} v\right\|_{H^{1}\left(\mathbb{R}_{+}^{2}\right)}^{2}+\frac{C^{\prime} L A}{2}\left\|D_{h} v\right\|_{L^{2}\left(\mathbb{R}_{+}^{2}\right)}^{2} \quad \forall A>0 \\
& \leq \frac{L}{2}\left(\frac{C}{A}+C^{\prime} A\right)\|\nabla v\|_{\left(L^{2}\left(\mathbb{H}_{+}^{2}\right)\right)^{2}}^{2}+\frac{C^{\prime} L}{2 A}\left\|\nabla D_{h} v\right\|_{\left(L^{2}\left(\mathrm{R}_{+}^{2}\right)\right)^{2}}^{2} \\
\quad \text { with Lemma translations }(v), & \\
& \quad \leq I_{1} \mid=\left\langle v D_{h} u, D_{h} v_{z}\right\rangle_{H^{1 / 2}(\mathbb{R}), H^{-1 / 2}(\mathbb{R})} \\
& \leq C\left\|\gamma\left(v D_{h} u\right)\right\|_{H^{1 / 2}(\mathbb{R})}\left\|D_{h} v\right\|_{H^{1}\left(\mathbb{R}_{+}^{2}\right)} .
\end{aligned}
$$

Remark that $\|\gamma v\|_{L^{\infty}} \leq L$ and $\left\|\gamma D_{h} u\right\|_{L^{\infty}} \leq L$; therefore one can prove that

$$
\left\|\gamma\left(v D_{h} u\right)\right\|_{H^{1 / 2}(\mathbb{R})} \leq L\|\gamma v\|_{H^{1 / 2}(\mathbb{R})}+I\left\|\gamma D_{h} u\right\|_{H^{1 / 2}(\mathbb{R})} \leq C_{L}\|v\|_{H^{1}\left(\mathbb{R}_{+}^{2}\right)}
$$

and, since this last norm is controlled,

$$
\left|I_{1}\right| \leq C_{1}\left\|\nabla D_{h} v\right\|_{\left(L^{2}\left(\boldsymbol{P}_{+}^{2}\right)\right)^{2}}+C_{1}^{\prime}
$$

Finally

$$
\begin{aligned}
& \forall A>0, \\
& \begin{aligned}
\left\|\nabla D_{h} v\right\|_{\left(L^{2}\left(\mathbb{R}_{+}^{2}\right)\right)^{2}}^{2} \leq & C_{1}\left\|\nabla D_{h} v\right\|_{\left(L^{2}\left(\mathbb{R}_{+}^{2}\right)\right)^{2}}+C_{2}(A)\|v\|_{H^{1}\left(\mathbb{R}_{+}^{2}\right)}^{2} \\
& +\frac{C_{3}}{A}\left\|\nabla D_{h} v\right\|_{\left(L^{2}\left(\mathbb{R}_{+}^{2}\right)\right)^{2}}^{2}+C_{4} .
\end{aligned}
\end{aligned}
$$

We choose $A=2 C_{3}$, thus $\left\|\nabla D_{h} v\right\|_{\left(L^{2}\left(\mathbb{R}_{+}^{2}\right)\right)^{2}}^{2}-2 C_{1}\left\|\nabla D_{h} v\right\|_{\left(L^{2}\left(\mathbb{R}_{+}^{2}\right)\right)^{2}}-C_{5} \leq 0$. Remark that all these constants are independent of $h$. 
It comes $\left\|\nabla D_{h} v\right\|_{\left(L^{2}\left(\mathbb{R}_{+}^{2}\right)\right)^{2}}^{2} \leq C$, which implies $v_{z} \in H^{1}\left(\mathbb{R}_{+}^{2}\right)$, then $\gamma u_{z}$ is in $H^{3 / 2}(\mathbb{R})$ and $\gamma u \in H^{5 / 2}(\mathbb{R})$. Therefore $\gamma u \in C^{1, \alpha}(\mathbb{R})$.

LEMma 3.5. - The solution $u$ of $(1.4 \mathrm{H})$ verifies $\left\|\gamma u_{z}\right\|_{L^{\infty}(\mathbb{R})}<\infty$.

Proof. - For $K \geq 0$ denote by $u^{K}$ the unique solution of $(1.4 \mathrm{H})$ corresponding to $K$ and $M(K)=\left\|\gamma u_{z}^{K}\right\|_{L^{\infty}(\mathbb{P})} \in[0,+\infty]$. Assume that there exists a $K_{1}>0$ such that $M\left(K_{1}\right)=+\infty$.

Define $K_{0}=\inf \left\{K \in \mathbb{R}_{+} / M(K)=+\infty\right\}$ : with the assumption on $K_{1}$ we have $K_{0} \leq K_{1} \leq+\infty$. Moreover, the implicit functions theorem applied to our system in a neighbourhood of ( $u=0, K=0)$ implies that for $K$ small enough the solution $u^{K}$ is very smooth (at least $C^{2, \alpha}$ ), so $u_{z}^{K}$ is bounded. Indeed, this function vanishes at the infinity since it is in $L^{2}$ and Hölder continuous. Therefore $K_{0}>0$.

Let $K<K_{0}$. For a given $K$, there holds $M(K)<\infty$, so Lemma 3.4 implies $u^{K} \in C^{1, \alpha}\left(\overline{\mathbb{R}_{+}^{2}}\right)$. We will now prove that $(M(K))_{K<K_{0}}$ is not bounded.

If this quantity is bounded by a constant $C_{0}$, then by the proof of Lemma 3.4, we have

$$
\left\|u^{K}\right\|_{C^{1, \alpha}\left(\overline{\left.\mathbf{R}_{+}^{2}\right)}\right.} \text { is uniformly bounded for } K<K_{0} .
$$

It follows that $u^{K} \stackrel{K \rightarrow K_{0}}{\longrightarrow} u^{K_{0}}$ in $C^{1, \alpha^{\prime}}\left(\overline{\mathbb{R}_{+}^{2}}\right)$ so $u^{K_{0}}$ is $C^{1, \alpha^{\prime}}$, where $\alpha^{\prime}<\alpha$. Hence $u^{K_{0}} \in C^{2, \alpha}\left(\overline{\mathbb{R}_{+}^{2}}\right)$ since it verifies now a regular elliptic boundary value problem. We can apply again the implicit functions theorem to $\left(u^{K_{0}}, K_{0}\right)$ to find that there exists $\eta>0$ such that on $] K_{0}-\eta, K_{0}+\eta$ [ the system $(1.4 \mathrm{H})$ has a regular $\left(C^{2, \alpha}\right)$ solution. In particular, its derivative is bounded; this leads to a contradiction with the definition of $K_{0}$, so $(M(K))_{K<K_{0}}$ is not bounded.

The remainder of the proof relies on a blowup technique. Define the sequence

$$
K_{n} \stackrel{n \rightarrow+\infty}{\longrightarrow} K_{0} \text { with } 0<K_{n}<K_{0}
$$

such that

$$
M\left(K_{n}\right) \stackrel{n \rightarrow+\infty}{\longrightarrow}+\infty
$$

Next we denote $\quad v_{n}=-u_{z}^{K_{n}}$

$$
\lambda_{n}=\left(M\left(K_{n}\right)\right)^{-1} \stackrel{n \rightarrow+\infty}{\longrightarrow} 0
$$

and $z_{n}$ such that $v_{n}\left(z_{n}, 0\right)=\max _{\mathbb{R}}\left(\gamma v_{n}\right)=M\left(K_{n}\right)$.

Vol. 16, n 2-1999. 
Remark that $v_{n} \rightarrow 0$ at the infinity (at least $C^{0, \alpha}$ and $L^{2}$ ) so $\left|z_{n}\right|<+\infty$.

We now rescale the function $v_{n}$ as follows:

$$
w_{n}(z, x)=\lambda_{n} v_{n}\left(z_{n}+\lambda_{n} z, \lambda_{n} x\right)
$$

We will prove the following assertions.

$$
\begin{aligned}
& \text { (i) } w_{n}(0,0)-1 \\
& \text { (ii) } w_{n} \longrightarrow 0 \text { in } H^{1}\left(\mathbb{R}_{+}^{2}\right) \text { weak } \\
& \text { (iii) } \exists C:\left\|\gamma w_{n}\right\|_{H^{3 / 2}(\mathbb{R})} \leq C .
\end{aligned}
$$

These three properties lead to a contradiction. Indeed with the Sobolev embeddings (iii) implies that a subsequence of $w_{n}$ converges to a limit $w$ in $C^{0, \alpha}$. Then with (i) and (ii) we have both $w \equiv 0$ and $w(0,0)=1$.

This contradiction shall end the proof :

$$
\forall K \geq 0, \quad M(K)<+\infty
$$

It now remains to prove the three assertions.

(i) is immediate.

(ii) comes from $\left\|v_{n}\right\|_{H^{1}\left(\mathbb{R}_{+}^{2}\right)} \leq\left\|u_{n}\right\|_{H^{2}\left(\mathbb{R}_{+}^{2}\right)} \leq C$ and

$$
\left\{\begin{array}{l}
\left\|w_{n}\right\|_{L^{2}\left(\mathbb{R}_{+}^{2}\right)}=\left\|v_{n}\right\|_{L^{2}\left(\mathbb{R}_{+}^{2}\right)} \leq C \\
\left\|\nabla w_{n}\right\|_{\left(L^{2}\left(\mathbb{R}_{+}^{2}\right)\right)^{\prime}}=\lambda_{n}\left\|\nabla v_{n}\right\|_{\left(L^{2}\left(\mathbb{R}_{+}^{2}\right)\right)^{2}} \leq C \lambda_{n} .
\end{array}\right.
$$

The key point that will imply (iii) is that $\max _{\mathbb{R}}\left(\gamma w_{n}\right)=1$ with this rescaling. We already know that $\left\|w_{n}\right\|_{H^{1}\left(\mathbb{R}_{+}^{2}\right)}$ is bounded but (iii) needs an estimate independent of $n$. It fact it is nearly the same as for Lemma 3.4: from a $L^{\infty}$ estimate of $\gamma w_{n}$ we obtain a $H^{3 / 2}(\mathbb{R})$ estimate of the same function, with a constant independent of $n$.

The proof is simpler here since, from Lemma 3.4, we know more regularity on $w_{n}$, at least that it is in $H^{2}\left(\mathbb{R}_{+}^{2}\right)$.

Let $\mu_{n}=\partial_{z} w_{n}$ (we change here the notation for the derivative because of the $n$, which is only an index). This function is in $H^{1}\left(\mathbb{R}_{+}^{2}\right)$, its $L^{2}\left(\mathbb{R}_{+}^{2}\right)$ norm is bounded by a constant independent of $n$ and it verifies

$$
\begin{cases}-\Delta \mu_{n}-\frac{\lambda_{n}^{2}}{2}\left(z \partial_{z} \mu_{n}+x \partial_{x} \mu_{n}\right)-\lambda_{n}^{2} \mu_{n}-\frac{\lambda_{n} z_{n}}{2} \partial_{z} \mu_{n}=0 & \left(\mathbb{R}_{+}^{2}\right) \\ \partial_{x} \mu_{n}=K_{n} \check{U}_{n} \partial_{z} \mu_{n}-3 K_{n} W_{n} \mu_{n} & (x=0)\end{cases}
$$


In the boundary term, $\quad \check{U}_{n(z, x)}=\left(u_{n}+\psi\right)_{\left(z_{n}+\lambda_{n} z, \lambda_{n} x\right)}$

$$
\begin{array}{ll} 
& W_{n(z, x)}=w_{n(z, x)}+\lambda_{n} \psi_{\left(z_{n}+\lambda_{n} z, \lambda_{n} x\right)} \\
\text { and } \quad & \mu_{n(z, x)}=\mu_{n(z, x)}+\lambda_{n}^{2} \psi_{z\left(z_{n}+\lambda_{n} z, \lambda_{n} x\right)} .
\end{array}
$$

Multiply by $\mu_{n}$, integrate by parts and estimate the different terms like for the proof of lemma 3.4. Remark that the contribution of the new term in the estimate is

$$
-\int_{\mathbb{R}_{+}^{2}} \frac{\lambda_{n} z_{n}}{2} \mu_{n} \partial_{z} \mu_{n}=0
$$

so we do not need to control $z_{n}$. It comes

$$
\left\|\nabla \mu_{n}\right\|_{\left(L^{2}\left(\mathbb{R}_{+}^{2}\right)\right)^{2}}^{2} \leq C
$$

Thus

$$
\begin{aligned}
\left\|\mu_{n}\right\|_{H^{1}\left(\mathbb{R}_{+}^{2}\right)} \leq C & \Longrightarrow\left\|\gamma \mu_{n}\right\|_{H^{1 / 2}(\mathbb{R})} \leq C^{\prime} \\
& \Longrightarrow\left\|\gamma w_{n}\right\|_{H^{3 / 2}(\mathbb{R})} \leq C^{\prime \prime}
\end{aligned}
$$

End of the proof of Theorem 1.1. - Due to Lemma 3.5, $\gamma u_{z} \in L^{\infty}(\mathbb{R})$. Hence with Lemma $3.4, u \in C^{1, \alpha}\left(\overline{\mathbb{R}_{+}^{2}}\right)$. Thus we have already seen that it implies $u \in C^{2, \alpha}\left(\overline{\mathbb{R}_{+}^{2}}\right)$.

Remark that because of the elliptic regularity, $u$ is already $C^{\infty}$ inside $\mathbb{R}_{+}^{2}$. We have to prove the smoothness on the boundary.

Write the system verified by $V=U_{z}$ :

$$
\begin{cases}-\Delta V-\frac{1}{2}\left(z V_{z}+x V_{x}\right)-\frac{1}{2} V=0 & \left(\mathbb{R}_{+}^{2}\right) \\ V_{x}=K U V_{z}-K V^{2} & (x=0) .\end{cases}
$$

Since we know that $U$ and $V$ are in $C^{1, \alpha}\left(\overline{\mathbb{R}_{+}^{2}}\right)$, this is a regular oblique derivative problem so $U_{z} \in C^{2, \alpha}\left(\overline{\mathbb{R}_{+}^{2}}\right)$. Hence the boundary condition of the system $(1.4 \mathrm{NH})$ verified by $U$, which is $U_{x}=K U U_{z}$, gives the $C^{2, \alpha}$ regularity of $U_{x}$ on the boundary.

Finally in this step we proved that $U \in C^{3, \alpha}\left(\overline{\mathbb{R}_{+}^{2}}\right)$. By successive derivations of $(1.4 \mathrm{NH})$, we obtain recurrently the $C^{\infty}$ regularity of $U$ on $\overline{\mathbb{R}_{+}^{2}}$. 


\section{CONVERGENCE TO SELF-SIMILAR SOLUTIONS}

A consequence of [7] is that problem $(1,1 \mathrm{NH})$ with, say, a $C^{2}$ initial datum, will have global classical solutions. To prove its convergence to a self-similar solution, we will first devise sub-and super-solutions to the problem in self-similar coordinates that will prove the uniform convergence. A stability argument will yield the convergence rate.

Recall that, if we now come back to the self-similar coordinates

$$
\tau=\log (t+1), \quad z=\frac{Z}{\sqrt{t+1}}, \quad x=\frac{X}{\sqrt{t+1}} .
$$

Problem (1.1NH) writes

$$
\begin{cases}B_{\tau}-\Delta B-\frac{1}{2}\left(z B_{z}+x B_{x}\right)=0 & \left(\mathbb{R}_{+}^{2}\right) \\ B_{x}-K B B_{z}=0 & (x=0) \\ B(\tau,-\infty, x)=1, \quad B(\tau,+\infty, x)=0 . & \end{cases}
$$

Recall also that any solution $\left(\psi_{\alpha}\right)_{\alpha \in \mathbb{R}}$ of

$$
\left\{\begin{array}{l}
-\psi_{\alpha}^{\prime \prime}(z)-\frac{1}{2} z \psi_{\alpha}^{\prime}(z)=0 \quad(z \in \mathbb{R}) \\
\psi_{\alpha}(-\infty)=1, \quad \psi_{\alpha}(+\infty)=\alpha
\end{array}\right.
$$

is a steady sub-solution to (4.1) as soon as $\alpha \leq 0$. Hence $\underline{B}(z)=$ $\max \left(\psi_{\alpha}, 0\right)$ is a steady sub-solution to (4.1). Remark that we impose the non-essential condition $B_{0} \geq 0$.

We now define a family of super-solutions that will be more flexible than the ones of Lemma 2.1 .

Proposition 4.1. - Let $B_{0}$ satisfy the assumptions of Theorem 1.2. There exists a steady super-solution to (4.1), denoted $\bar{B}$, such that $B_{0}(z, x) \leq$ $\bar{B}(z, x)$.

Proof. - . What prevents us from using Lemma 2.1 is that $B_{0}$ may be of arbitrary size at finite distance. We thus look for a super-solution $\bar{B}$ to (4.1) under the form

$$
\begin{gathered}
\bar{B}(z, x)=\psi(z)+2 \psi(x) h(z), \\
h(z)= \begin{cases}\lambda_{1}(1-\psi(z)) & \text { for } z \leq 0, \\
\lambda_{1}(1-\psi(z))-a z & \text { for } 0 \leq z \leq z_{1}, \\
\lambda_{2} \psi\left(z-z_{0}\right) & \text { for } z_{1} \leq z,\end{cases}
\end{gathered}
$$


where the parameters $\lambda_{1}, \lambda_{2}, a, z_{0}, z_{1}$ are to be chosen. We impose the continuity of $\bar{B}$ :

$$
\lambda_{1}\left(1-\psi\left(z_{1}\right)\right)-a z_{1}=\lambda_{2} \psi\left(z_{1}-z_{0}\right)
$$

If $a$ is nonnegative, this implies, as in Lemma 2.1, that to satisfy the first inequality of (2.2) we only need to have

$$
h^{\prime}\left(z_{1}^{+}\right)-h^{\prime}\left(z_{1}^{-}\right) \leq 0
$$

a sufficient condition for this is

$$
a \leq \frac{\lambda_{2}}{2 \sqrt{\pi}} \exp -\frac{\left(z_{1}-z_{0}\right)^{2}}{4} .
$$

The boundary inequality, $\bar{B}_{x} \leq K \overline{B B}_{z}$, is fulfilled as follows.

- For $z \leq 0$, there only needs to hold $\lambda_{1} \geq 1$.

- For $0 \leq z \leq z_{1}$, after direct computations, the condition writes

$$
a+\left(\lambda_{1}-1\right) \psi^{\prime}(z) \leq \frac{1}{K \sqrt{\pi}}\left(1-\frac{\psi(z)}{\lambda_{1}(1-\psi(z))-a z+\psi(z)}\right)
$$

this inequality is verified if $a$ is lower than its right-hand side, since $\psi^{\prime} \leq 0$. One can see that, since $\psi$ is convex on $\mathbb{R}_{+}$, this right-hand side is larger than its values in 0 and in $z_{1}$, which are respectively $\frac{1}{K \sqrt{\pi}} \frac{\lambda_{1}}{1+\lambda_{1}}$ and, with [C1], $\frac{1}{K \sqrt{\pi}}\left(1-\frac{\psi\left(z_{1}\right)}{\psi\left(z_{1}\right)+\lambda_{2} \psi\left(z_{1}-z_{0}\right)}\right) \geq \frac{1}{K \sqrt{\pi}} \frac{\lambda_{2}}{1+\lambda_{2}}$. Hence, if $\lambda_{1}>1$ and $\lambda_{2}>1$, it is sufficient to have

$$
a \leq \frac{1}{2 K \sqrt{\pi}}
$$

- For $z_{1} \leq z$, the condition is fulfilled if, and only if,

$$
\frac{K}{2}\left(\lambda_{2} e^{-\left(z-z_{0}\right)^{2} / 4}+e^{-z^{2} / 4}\right)\left(1+\frac{\psi(z)}{\lambda_{2} \psi\left(z-z_{0}\right)}\right) \leq 1,
$$

which, if $K \frac{\left(1+\lambda_{2}\right)^{2}}{2 \lambda_{2}}>1$, is implied by

$$
z_{1} \geq z_{0}+2 \sqrt{\log \left(K \frac{\left(1+\lambda_{2}\right)^{2}}{2 \lambda_{2}}\right)}
$$

The last property that $\bar{B}$ has to verify to be a super-solution is $\bar{B} \geq B_{0}$. 
Now, let $B_{0}$ satisfy the assumptions of 'Theorem 1.2: $b_{0}(z, x)=$ $B_{0}(z, x)-\psi(z)$ is small enough at the infinity. First, one fixes the parameters $\lambda_{1}>\lambda_{2}>1$ such that $b_{0}(z, x) \leq 2 \psi(x) \cdot \lambda_{1}(1-\psi(z))$ for $z \leq 0$. $x \geq 0$, and $b_{0}(z, x) \leq 2 \psi(x) \cdot \lambda_{2} \psi(z)$ for $z \geq 0$. $x \geq 0$. Next, if moreover $\lambda_{2}$ then $\lambda_{1}$ have been chosen large enough, we let

$$
\begin{gathered}
z_{1}=4 K \sqrt{\pi} \lambda_{1}, \\
z_{0}=z_{1}-2 \sqrt{\log \left(K \frac{\left(1+\lambda_{2}\right)^{2}}{2 \lambda_{2}}\right)} \geq 0 ;
\end{gathered}
$$

[C4] is fulfilled. We can now define $a$ by [Cl]:

$$
a=\frac{1}{4 K \sqrt{\pi}}\left(1-\psi\left(z_{1}\right)-\frac{\lambda_{2}}{\lambda_{1}} \psi\left(z_{1}-z_{0}\right)\right) \leq \frac{1}{4 K \sqrt{\pi}} ;
$$

there holds $a \geq 0$ since $\psi(z) \leq 1 / 2$ for $z \geq 0$. Moreover [C2] and [C3] are satisfied.

It remains to verify [C5]. For $z \leq 0$ the super-solution lies already above $B_{0}$, thanks to the choice of $\lambda_{1}$. For $z \geq z_{1}$, we just remark that $B_{0} \leq \psi(z)+2 \psi(x) \lambda_{2} \psi(z) \leq \psi(z)+2 \psi(x) \lambda_{2} \psi\left(z-z_{0}\right)=\bar{B}(z, x)$.

Finally, for $0 \leq z \leq z_{1}$, a simple study of the function $\lambda_{1}(1-\psi(z))-a z-$ $\lambda_{2} \psi(z)$ shows that it is larger than its values in 0 and $z_{1}$, which are positive. Therefore $B_{0} \leq \psi(z)+2 \psi(x) \lambda_{2} \psi(z) \leq \bar{B}(z, x)$ and [C5] is fulfilled.

Remark that when we let $\lambda_{1}=\lambda_{2}=1$ in the above formulae, the parameters $a, z_{0}$ and $z_{1}$ are the same as in Lemma 2.1.

Therefore we have the first part of Theorem 1.2, expressed in the self-similar variables:

Corollary 4.2. - There holds

$$
\lim _{\tau \rightarrow+\infty}\|B(\tau)-U\|_{\infty}=0
$$

where $U(z, x)$ is the self-similar solution of $(1.4 \mathrm{NH})$.

Proof. - It only remains to remark that, if $B_{0}$ satisfies the assumptions of Theorem 1.2, then there is $\alpha \geq 0$ such that $B:=\max \left(\psi_{c}, 0\right) \leq B_{0}$. Therefore $B_{0}$ is between a steady sub-solution and a steady super-solution of (4.1), and the convergence result follows just as in Section 2, using the method of Sattinger [16]. We just give a sketch of this proof.

Let $\overline{\mathcal{B}}(\tau, z, x)$ (resp. $\underline{B}(\tau, z, x)$ ) be the solution of $(4.1)$ with $\bar{B}(z, x)$ (resp. $\underline{B}(z)$ ) as an initial datum. These functions are defined as follows. 
$\bar{B}$ fulfills the assumptions of Theorem 1.2 , since $\bar{B}(z, x)-\psi(z) \rightarrow 0$ as $|(z, x)| \rightarrow \infty$, so it enables us to define $\overline{\mathcal{B}}(\tau, z, x)$ directly as the solution of (4.1) in the sense explained at the beginning of Section $2: \bar{B}-\psi$ is the solution of the homogeneous associated problem. On the other hand, $\underline{B}$ does not necessarily tend to $\psi$ at the infinity. Nevertheless, we define $\underline{\mathcal{B}}(\tau, z, x)$ as follows. Let $\underline{\mathcal{B}}^{\infty}(\tau, z)$ be the solution of the equation

$$
\left\{\begin{array}{l}
\underline{\mathcal{B}}_{\tau}^{\infty}-\underline{\mathcal{B}}_{z z}^{\infty}-\frac{1}{2} z \underline{\mathcal{B}}_{z}^{\infty}=0(\tau>0, z \in \mathbb{R}) \\
\underline{\mathcal{B}}^{\infty}(\tau,-\infty)=1, \quad \underline{\mathcal{B}}^{\infty}(\tau,+\infty)=0 \\
\underline{\mathcal{B}}^{\infty}(0, z)=\underline{B}(z)
\end{array}\right.
$$

Then $\underline{\mathcal{B}}(\tau, z, x)$ is defined as the solution of $(4.1)$ such that $\underline{\mathcal{B}}(\tau, z, x)-$ $\underline{\mathcal{B}}^{\infty}(\tau, z) \rightarrow 0$ as $|(z, x)| \rightarrow \infty$.

One proves that $\overline{\mathcal{B}}(\tau, z, x)$ is decreasing in time, that $\underline{\mathcal{B}}(\tau, z, x)$ is increasing, and that both functions converge uniformly to the self-similar solution $U(z, x)$ as $\tau \rightarrow+\infty$ (remark that $\underline{\mathcal{B}}^{\infty}(\tau, z) \rightarrow \psi$ ). Finally, since for all time there holds $\underline{\mathcal{B}}(\tau) \leq B(\tau) \leq \overline{\mathcal{B}}(1)$, the result follows.

Let us turn to the exponential convergence with respect to $\tau$. Let $L$ be the differential operator

$$
L=-\Delta-\frac{1}{2}\left(z \frac{\partial}{\partial z}+x \frac{\partial}{\partial x}\right) .
$$

No domain will be specified, due to the form of the boundary conditions. In particular, we do not know whether a Krein-Rutman-type principle applies for $L$ in the natural weighted space where we would like to carry out our study. The following lemma, which initiates the asymptotic behaviour of $U$, will be useful.

LEMMA 4.3. - There holds

$$
\frac{\left|U_{z z}\right|}{-U_{z}}+\frac{\left|U_{z x}\right|}{-U_{z}} \leq C_{1} e^{C_{2}(|x|+|z|)} \quad \text { as }(|z, x|) \rightarrow \infty .
$$

Proof. - Theorem 1.1 tells us that the equation for $V:=U_{z}$ may be handled as a regular boundary problem that we write

$$
\begin{cases}-\Delta V-\frac{1}{2} V+f_{1}=0 & \left(\mathbb{R}_{+}^{2}\right) \\ V_{x}-f_{2} V_{z}-f_{3} V-0 & (x-0) \\ V(-\infty, x)=0, \quad V(+\infty, x)=0 & \\ V(z,+\infty)=-\psi_{z}, & \end{cases}
$$


where the $C^{\infty}$ functions are $f_{1}=-\frac{1}{2}\left(x V_{z}+z V_{z}\right), f_{2}=K U, f_{3}=K V$. Hence elliptic estimates [1] and interpolation lead to the following estimates. Let $K(\alpha, x, z)$ denote the intersection of $\mathbb{R}_{+}^{2}$ with the cube centered at $(x, z)$ and with length $\alpha$; we have

$$
\left\|U_{z z}\right\|_{L^{\infty}(K(1, x, z))}+\left\|U_{z x}\right\|_{L^{x}(K(1, x, z))} \leq C(|x|+|z|)\left\|U_{z}\right\|_{L^{\infty}(K(2, x, z)\}} .
$$

We now come back to the usual writing of the elliptic equation for $U_{z}$ : the Harnack inequalities are applicable to it and - Berestycki, Caffarelli, Nirenberg [4] - they can be pushed to the boundary of $\mathbb{R}_{+}^{2}$ due to the particular form of the boundary condition, which allows an extension of $U$. along the lines of the vector field $(1,-K U)$. Inspection of the Harnack constant - see, for example, Trudinger [17], Theorem 1.1 - reveals that it is no larger than $e^{O(|x|+|z|)}$; hence there exists a constant $C$ such that

$$
\left\|U_{z}\right\|_{L^{\times}(h(2, n z))} \leq C e^{C(|x|+|:|)} \min _{K(2, r, *)}\left(-U_{z}\right) .
$$

This is enough to get estimate 4.3.

End of the proof of Theorem 1.2. - Set

$$
B(\tau, z, x)=U(z, x)+U_{z}(z, x) v(\tau, z, x) .
$$

Remark that since we already know that $U_{2} \leq 0$ and is smooth (Theorem 1.1), the maximum principle and Hopf's Lemma imply that $U_{z}$ does not vanish on $\overline{\mathbb{R}_{+}^{2}}$. Indeed, consider for that the equations on $w=-U_{z}$, and put the zero-order term -whose coefficient has the bad signin the right-hand side of the elliptic equation.

The function $v$ verifies

$$
\begin{cases}v_{\tau}+L v-2 \frac{\nabla U_{z}}{U_{z}} \cdot \nabla v+\frac{1}{2} v=0 & \left(\mathbb{R}_{+}^{2}\right) \\ v_{x}=K\left(U+U_{z} v\right) v_{z}+K U_{z} v^{2} . & (x=0)\end{cases}
$$

The zero-order term of the equation inside $\mathbb{R}_{+}^{2}$ has a positive coefficient (1/2) which will provide the convergence rate thanks to the maximum principle. Nevertheless, one cannot use this principle directly on the function $v$ because of the boundary term: indeed, the sign of $U_{z z} v$, thc cocfficicnt of $v$ in its right-hand side, is not necessarily positive. That is the reason why we shall introduce another function $w$.

For that, let $\mu, \lambda$ be two positive real number, to be precised further. We define the function $\nu(z)$ by a regularization (near zero) of $\exp (-\lambda|z|)$ and $\Gamma$ by

$$
\Gamma(z, x)=x \exp (-\lambda x) \nu(z)
$$


We set now

$$
v(\tau, z, x)=(1-\mu \Gamma(z, x)) w(\tau, z, x)
$$

this new function $w$ satisfies

$$
\begin{cases}w_{\tau}+L w+\mathbf{P}(z, x) \cdot \nabla w+\left(\frac{1}{2}+\mu g(z, x)\right) w=0 & \left(\mathbb{R}_{+}^{2}\right) \\ w_{x}=h w_{z}+f w, & (x=0)\end{cases}
$$

where

$$
\begin{gathered}
\mathbf{P}(z, x)=-2 \frac{\nabla U_{z}}{U_{z}}+2 \mu \frac{\nabla \Gamma}{1-\mu \Gamma}, \\
g(z, x)=-(1-\mu \Gamma)^{-1}\left(L \Gamma-2 \frac{\nabla U_{z}}{U_{z}} \cdot \nabla \Gamma\right), \\
h=K\left(U+U_{z}(1-\mu \Gamma) w\right), \\
f(\tau, z)=K U_{z z}(z, 0) v(\tau, z, 0)+\mu \nu(z) .
\end{gathered}
$$

To apply the maximum principle to $w$, we necd to choose the different parameters such that $\mu g$ is small and $f$ is positive.

Let $\alpha$ be a positive small real number. We fix $\lambda=2 C_{2}$, where $C_{2}$ is the same as in the statement of Lemma 4.3: thanks to the form of $\Gamma$ and to this Lemma, $\nabla \Gamma . \nabla U_{z} / U_{z}$ is uniformly bounded on $\mathbb{R}_{+}^{2}$. Since $L \Gamma$ is also bounded, it suffices to choose $\mu$ small enough to ensure $|\mu g(z, x)|<\alpha$ on $\mathbb{R}_{+}^{2}$. We fix here this constant $\mu$.

Now, in the boundary term, we write $K U_{z z} v=K \frac{U_{z z}}{U_{z}}(B-U)$ and, thanks to the sub-and super-solutions, their exists a constant $C$ such that

$$
\forall(t, z) \in \mathbb{R}_{+} \times \mathbb{R} \quad|B(t, z, 0)-U(z, 0)| \leq C \exp \left(-\frac{z^{2}}{8}\right) ;
$$

hence, since $\nu(z) \underset{\infty}{\sim} \exp (-\lambda z)$ and with Lemma 4.3 , one can fix $Z_{0}$ such that

$$
\forall \tau>0, \forall|z|>Z_{0}, \quad\left|K U_{z z} v\right|(\tau, z, 0)<\mu \nu(z): \quad f(\tau, z)>0 .
$$

Let $\delta=\min _{\left[-Z_{0}, Z_{0}\right]}(\nu)>0$ and $M=\max _{\left[-Z_{0}, Z_{0}\right]}\left|U_{z z} / U_{z}\right|$. By Corollary 4.2 one can now find a $\tau_{c}>0$ such that, for $\tau>\tau_{c}$, for $(z, x) \in \mathbb{R}_{+}^{2}$, $|B-U|(\tau, z, x)<\frac{\mu \delta}{K M}$. This implies

$$
\forall \tau>\tau_{c}, \forall|z| \leq Z_{0}, \quad K\left|\frac{U_{z z}}{U_{z}}(B-U)\right|<\mu \nu: \quad f(\tau, z)>0 .
$$


Finally, remark that the Harnack inequalities applied (as in Lemma 4.3) on the function $-U_{z}$ imply that the increase of $w$ at the infinity is bounded by $C e^{C\left(z^{2}+x^{2}\right)}$. Therefore, with this set of parameters $\left(\mu, \lambda, \tau_{c}\right)$, the maximum principle applies for $\tau>\tau_{r}$. and yields

$$
w(\tau, z, x) \leq\left\|w\left(\tau_{\delta}\right)\right\|_{\infty} e^{-(1 / 2-\alpha) \tau}
$$

The same argument may be used to find a lower bound for $w$. The result follows if we come back to the variable $t$, and the function $B-U$.

Remark. - Application of the classical regularity results [11], [12] yields the exponential convergence in $C^{2}$.

\section{ACKNOWLEDGEMENTS}

The authors would like to thank Prof. P.-A. Raviart for having suggested them this work and for his encouragements during its preparation.

\section{REFERENCES}

[1] S. Agmon, A. Douglis and L. Nirenberg, Estimates near the boundary for solutions of elliptic partial differential equations satisfying general boundary conditions I and II. Comm. Pure Appl. Math., Vol. 12, 1959, pp. 623-727; Vol. 17, 1964, pp. 35-92.

[2] H. Amann, Quasilinear Parabolic Systems under Nonlinear Boundary Conditions, Arch. Rat. Mech. Anal., Vol. 92, 1986, pp. 153-191.

[3] H. AmanN, Parabolic: Evolution Equations and Nonlinear Boundary Conditions, J. Diff. Eq., Vol. 72, 1988, pp. 201-269.

[4] H. Berestycki, L. A. Caffarelli and L. Nirenberg, Uniform estimates for regularizations of free boundary problems, Analysis and partial differential equations, C. Sadosky \& M. Dekker eds., 1990, pp. 567-617.

[5] H. BRÉzIS, Analyse Fonctionnelle dans Mathématiques appliquées pour la maîtrise, Masson. 1983.

[6] A. Chuvatın, Thèse, Ecole Polytechnique, 1994.

[7] G. DONG, Initial and nonlinear oblique boundary value problems for fully nonlinear parabolic equations, J. Partial Diff. Eq., Vol. 1, 1988, pp. 12-42.

[8] A. V. GordeEv, A. V. Grechikha and Y. L. KALDA, Rapid penetration of a magnetic field into a plasma along an electrode, Sov. J. Plasma Phys., Vol. 16, 1, 1990.

[9] A. V. GoRdEEV, A. S. KingSEP and L. I. RudaKov, Electron Magnetohydrodynamics, Physics Reports, Vol. 243, 5, 1994.

[10] A. S. Kingsep, K. V. Chukbar and V. V. Yan ${ }^{-k o v, ~ R e v i e w s ~ o f ~ P l a s m a ~ P h y s i c s, ~ V o l . ~} 16$. B. B. Kadomtsev (ed), Plenum, New-York, 1990.

[11] O. A. Ladyženskaja, V. A. Solonnikov and N. N. Ural ceva, Linear and quasilinear equations of parabolic type, Transl. Math. Monographs, Vol. 23, Amer. Math. Soc.. Providence R.I., 1968.

[12] O. A. LADYŽEnSKaja and N. N. URAL'CEVA, Équations aux dérivées partielles de type elliptique, Monogr. Univ. Math., Dunod, Paris, 1968.

[13] G. LiEBERman and N. TrudingER, Nonlinear oblique boundary value problems for nonlinear elliptic equations, Trans. A.M.S, Vol. 295, 1986, pp. 509-546. 
[14] Г. MÉHATS and J.-M. RoQuejoffre, A nonlinear oblique derivative boundary value problem for the heat equation, Part II: rapid penetration at the boundary, to appear.

[15] A. I. Nazarov and N. N. Ural TSeva, A Problem with an Oblique Derivative for a Quasilinear Parabolic Equation, Zap. Nauch. Sem. S-Peterburg Otdel. Mat. Inst. Steklov (POMI), Vol. 200, 1992.

[16] D. H. Sattinger, Monotone Methods in Nonlinear Elliptic and Parabolic Boundary Value Problems, Indiana University Mathematics Journal, Vol. 21, n" 11, 1972, pp. 979-1000.

[17] N. TRUDINGER, On Harnack type inequalities and their applications to quasilinear problems. Comm. Pure Appl. Math., Vol. 20, 1967, pp. 721-747.

(Manuscript received February 20, 1907.) 